\title{
X-RAY GALAXY CLUSTERS IN NOSOCS: SUBSTRUCTURE AND THE CORRELATION OF OPTICAL AND X-RAY PROPERTIES
}

\author{
P. A. A. Lopes, R. R. de Carvalho, and H. V. Capelato \\ Instituto Nacional de Pesquisas Espaciais-Divisão de Astrofísica, Avenida dos Astronautas, 1758, \\ São José dos Campos, SP 12227-010, Brazil; paal@das.inpe.br \\ R. R. GAL \\ University of California, Department of Physics, One Shields Avenue, Davis, CA 95616 \\ S. G. DJORGOVSKI \\ Palomar Observatory, Caltech, MC 105-24, Pasadena, CA 91125 \\ R. J. BRUNNER \\ University of Illinois, Department of Astronomy, 1002 West Green Street, Urbana, IL 61801 \\ S. C. ODEWAHN \\ Hobby-Eberly Telescope, HC75-Box 1337-10, Fort Davis, TX 79734-5015
}

AND

A. A. Mahabal

Palomar Observatory, Caltech, MC 105-24, Pasadena, CA 91125

Received 2006 February 14; accepted 2006 May 5

\begin{abstract}
We present a comparison of optical and X-ray properties of galaxy clusters in the northern sky, using literature data from BAX and optically selected clusters in DPOSS. We determine the recovery rate of X-ray-detected clusters in the optical as a function of richness, redshift, and X-ray luminosity, showing that the missed clusters are typically lowcontrast systems when observed optically (either poor or at high redshifts). We employ four different statistical tests to test for the presence of substructure using optical two-dimensional data. We find that approximately $35 \%$ of the clusters show strong signs of substructure in the optical. However, the results are test-dependent, with variations also due to the magnitude range and radius utilized. We have also performed a comparison of X-ray luminosity and temperature with optical galaxy counts (richness). We find that the slope and scatter of the relations between richness and the X-ray properties are heavily dependent on the density contrast of the clusters. The selection of substructurefree systems does not improve the correlation between X-ray luminosity and richness, but this comparison also shows much larger scatter than one obtained using the X-ray temperature. In the latter case, the sample is significantly reduced because temperature measurements are available only for the most massive (and thus high-contrast) systems. However, the comparison between temperature and richness is very sensitive to the exclusion of clusters showing signs of substructure. The correlation of X-ray luminosity and richness is based on the largest sample to date ( 750 clusters), while tests involving temperature use a similar number of objects as previous works $(\lesssim 100)$. The results presented here are in good agreement with existing literature.
\end{abstract}

Subject headings: catalogs — galaxies: clusters: general $-\mathrm{X}$-rays: galaxies

Online material: color figures

\section{INTRODUCTION}

In most scenarios of structure formation, galaxy clusters are the largest and latest objects to form under the influence of their own gravity. Reliable measurements of cluster masses allow us to study the evolution of the large-scale structure through the cluster mass function and its time evolution. Several studies have examined the evolution experienced by clusters, using the results to provide estimates of important cosmological parameters, such as $\Omega_{m}$ and $\sigma_{8}$ (Eke et al. 1998; Mathiesen \& Evrard 1998; Kitayama \& Suto 1997; Bahcall et al. 1997, 2003; Carlberg et al. 1997; Donahue et al. 1998; Reichart et al. 1999; Blanchard \& Bartlett 1998; Postman et al. 2002). The main difficulty faced by these studies lies in determining the cluster masses. A variety of techniques can be used for this measurement, including dynamical methods (velocity dispersions), measuring the temperature of the intracluster gas, observations of the Sunyaev-Zel'dovich effect, and weak gravitational lensing. All of these methods are obser- vationally expensive, which render large-scale structure studies based on extensive samples impractical.

An alternative method to obtain mass estimates for a large sample of clusters is to connect the cluster mass to an easily observable parameter, such as X-ray luminosity $\left(L_{\mathrm{X}}\right)$ or the number of constituent galaxies (richness). The tightest mass-observable relations involve X-ray temperatures $\left(T_{\mathrm{X}}\right)$ and spectroscopically measured velocity dispersions $\left(\sigma_{v}\right)$. Unfortunately, large samples of clusters with either or both of these parameters measured do not exist currently. The X-ray luminosity $\left(L_{\mathrm{X}}\right)$ and optical richness (or luminosity, $L_{\text {opt }}$ ) also correlate well with mass, although with larger scatter than relations with $T_{\mathrm{X}}$ and $\sigma_{v}$. The precise measurement and calibration of these relations are essential for reliable mass function estimates. For instance, underestimation of the scatter in the mass-observable relation can result in overestimation of $\sigma_{8}$ (Voit 2005). Furthermore, the correlation of mass to properties determined at different wavelengths can lead to contradictory results, which may be associated with the selection function of the 
cluster catalogs constructed in different regimes, or with physical processes and evolution within the cluster populations.

Thus, understanding the systematics present in optical and X-ray surveys, and the way they complement each other, are necessary precursors to studies of cluster evolution and are even more important for cosmological tests that require statistically robust cluster samples. Some of these issues have been addressed in recent literature, either by comparing X-ray and optical cluster catalogs or conducting joint X-ray/optical surveys of galaxy clusters (Donahue et al. 2001, 2002; Gilbank 2001, 2004; Yee \& Ellingson 2003; Basilakos et al. 2004; Popesso et al. 2004, 2005; Smith et al. 2005). A very important by-product of such comparisons is the selection and follow-up study of unusual clusters, such as those that are optically rich but X-ray-underluminous (Gilbank 2001; Gilbank et al. 2004; Lubin et al. 2004).

As mentioned above, an important issue concerning these scaling relations is the precision to which they can be established and which observational biases contribute to increasing their scatter. Information on the distribution of galaxies, hot gas, and dark matter within clusters can play an important role in understanding this scatter. The diversity in the dynamical states of galaxy clusters may be associated with the uncertainties in the determination of the scaling relations. The presence of substructure is a clear sign of incomplete relaxation in a cluster, and can be estimated from the X-ray emission from the intracluster medium, as well as the distribution (one-, two-, or three-dimensional) of cluster galaxies (Mathiesen et al. 1999; Kolokotronis et al. 2001; Smith et al. 2005). The dynamical state of galaxy clusters is also strongly related to the underlying cosmology, so that the evolution of cluster substructure with look-back time is a powerful tool for constraining cosmological parameters (Mohr et al. 1995).

A few recent works have investigated the possibility that cluster substructure adds scatter to the observed scaling relations, but the results have been ambiguous. While Smith et al. (2005) find that the observational scaling relations of clusters behave differently for relaxed and unrelaxed clusters, O'Hara et al. (2006) find the opposite (based both on simulations and observational data). Some of these differences may be due to the relatively small sample size or to the inconsistent techniques for measuring substructure.

The main goal of this paper is the establishment of scaling relations for galaxy clusters through the comparison of optical and X-ray properties ( $N_{\text {gals }}, L_{\mathrm{X}}$, and $\left.T_{\mathrm{X}}\right)$. We also want to examine which factors contribute to the observed scatter in these relations. We first investigate the recovery rate of X-ray-emitting galaxy clusters in an optical survey, searching specifically for X-ray-detected clusters that could be missed in the optical. We also conduct a substructure study based solely on the photometric data. Finally, we compare the optical and X-ray properties of galaxy clusters, investigating the dependence of the results with the contrast cut of the sample used, the centroid adopted for the richness measures, and the amount of substructure. We limit this study to photometric and X-ray parameters and investigate the systematics in these comparisons, postponing to a future work the extension of the cluster scaling relations for other parameters, such as $R_{200}, \sigma_{v}$, and especially mass.

We use a list of X-ray-observed galaxy clusters from BAX (Base de Données Amas de Galaxies X) as our basis. ${ }^{1}$ The catalog initially comprises 914 galaxy clusters in the northern hemisphere, spanning the redshift range $0.05 \leq z \leq 0.40$. We trim this list to the same area covered by the Northern Sky Optical Cluster Survey (NoSOCS) in order to compare the two catalogs.

1 See http://bax.ast.obs-mip.fr.
The final X-ray list contains 792 clusters, of which $638(81 \%)$ are detected in the optical.

The remainder of this paper is organized as follows. In $\S 2$ we describe the X-ray data, while in $\S 3$ we provide details about the NoSOCS catalog. The BAX and NoSOCS comparison is discussed in $\S 4$, while the substructure analysis is presented in $\S 5$. In $\S 6$ we investigate the correlation between optical and X-ray properties, namely, $N_{\text {gals }}, L_{\mathrm{X}}$, and $T_{\mathrm{X}}$. The X-ray temperatureluminosity relation is also determined. In particular we investigate the impact of substructure and the cluster contrast on the scatter of these relations. We summarize our results in $\S 7$. We use $H_{0}=100 h \mathrm{~km} \mathrm{~s}^{-1} \mathrm{Mpc}^{-1}$, except where the $h$-dependence is explicitly given, and $q_{0}=0.5$ ( $h$ is fixed to 0.5 only for converting magnitudes, but not for computing angular sizes). In order to be in agreement with $\mathrm{BAX},{ }^{2}$ we decided not to adopt the currently favored cosmology with $h=0.70, \Omega_{m}=0.30$, and $\Omega_{\Lambda}=0.70$. This choice has no significant effect on the results for the scaling relations (namely, slope, intercept, and scatter). The only parameters that might be affected by changing cosmology are those associated with evolution, which is not expected to be significant in the current work due to the small redshift range probed.

\section{X-RAY DATA}

We search for optical counterparts of X-ray-observed clusters selected from BAX, which is an online research database containing information on all galaxy clusters with X-ray observations to date. BAX contains all galaxy clusters confirmed as X-ray sources, but it is not restricted to objects first detected in X-rays, and includes optically selected clusters (such as Abell clusters) with X-ray follow-up. The database allows us to retrieve each cluster's coordinates and redshift; various X-ray observational measurements, namely, flux $\left(F_{\mathrm{X}}\right)$, luminosity $\left(L_{\mathrm{X}}\right)$, and temperature $\left(T_{\mathrm{X}}\right)$; and a set of bibliographical references. The cluster coordinates and redshift are automatically generated from the NASA/ IPAC Extragalactic Database (NED). ${ }^{3} F_{\mathrm{X}}$ and $L_{\mathrm{X}}$ are in the ROSAT $[0.1-2.4 \mathrm{keV}]$ band. $F_{\mathrm{X}}$ and $L_{\mathrm{X}}$ units are $10^{-12} \mathrm{ergs} \mathrm{s}^{-1}$ $\mathrm{cm}^{-1}$ and $10^{44} \mathrm{ergs} \mathrm{s}^{-1}$, respectively. Only BAX canonical values of $F_{\mathrm{X}}, L_{\mathrm{X}}$, and $T_{\mathrm{X}}$ are retrieved. These are values chosen among the most precise and/or latest published measurements, meaning that if there are multiple references for a given cluster, the most precise and/or recent is kept. The BAX output is given for $H_{0}=50 \mathrm{~km} \mathrm{~s}^{-1} \mathrm{Mpc}^{-1}$ and $\Omega_{m}=1.0$. At the time of our query, BAX contained information on 1851 groups and clusters of galaxies, with 1656 clusters having luminosity measurements and 463 clusters with temperature measurements.

We performed a multicriterion search with the following constraints: $-5^{\circ} \leq \delta \leq 90^{\circ}$ and $0.05 \leq z \leq 0.40$. These limits ensure overlap with our optical galaxy cluster catalog (NoSOCS), which is based on the Palomar Digital Sky Survey (DPOSS; $\delta>-2^{\circ} .5$ and $z \lesssim 0.3$ ). The query was performed on 2005 April 12 , resulting in a list of 914 galaxy clusters. We then compared the X-ray centers of these clusters to all the plate limits used for NoSOCS, keeping only those that fall within these limits. We found 800 X-ray clusters within the NoSOCS limits, from which we eliminated eight that overlapped with bad areas from our survey (excised regions due to saturated objects such as bright stars). Of these 792 remaining systems, 638 have an optical counterpart in our catalog. From these 638 common clusters, 620 have measured X-ray luminosities (with two others discarded due to problems

\footnotetext{
${ }^{2}$ See http://bax.ast.obs-mip.fr/html/help/bax_help.html for notes on the BAX data.

3 See http://nedwww.ipac.caltech.edu.
} 
measuring the optical richness), while 101 have X-ray temperatures determined. More details on the recovery rate of X-ray galaxy clusters by DPOSS are provided in $\S 4$. The comparison of optical and X-ray properties, shown in $\S 6$, is based on this list of 618 common clusters with available $L_{\mathrm{X}}$ and $N_{\text {gals }}$, or the subset of 101 clusters for which temperatures are available.

\section{OPTICAL DATA}

The X-ray galaxy cluster catalog derived from BAX is compared to the NoSOCS (Gal et al. 2003; R. R. Gal et al. 2006, in preparation [Papers II and III, respectively]), which is a catalog of galaxy clusters constructed from the digitized version of the Second Palomar Observatory Sky Survey (POSS-II; DPOSS). NoSOCS is derived from high-latitude fields $|b|>30^{\circ}$, covering $\sim 11,000 \mathrm{deg}^{2}$ and containing $\sim 16,000$ cluster candidates. Richness and redshift estimates are provided for nearly all clusters; the richness measure (denoted $N_{\text {gals }}$ ) is described in detail in Gal et al. (2003) and in the next section. The median redshift of the sample is $z_{\text {med }}=0.16$, with a median richness of $N_{\text {gals,med }}=31$. From the selection functions presented in Papers II and III we expect to find rich clusters $\left(N_{\text {gals }} \sim 100\right)$ out to $z \sim 0.3$. This is the largest galaxy cluster catalog available to the present date. ${ }^{4}$

\section{RICHNESS AND THE BAX-NOSOCS COMPARISON}

Due to differences between the photometric redshift estimates from NoSOCS and the spectroscopic redshifts provided by BAX, we adopt the BAX redshift for all analysis involving clusters common to the two data sets. We note that the scatter in the comparison of NoSOCS and BAX redshifts is comparable to the redshift accuracy estimated by Gal et al. (2003). We utilize the BAX redshifts measures as they are generally more accurate (as they are typically spectroscopic), and the richness measure and its accuracy are sensitive to the cluster centroid, radius, and redshift adopted. This choice is corroborated by the fact that the comparisons of optical and X-ray properties show reduced scatter when using the BAX redshifts (described in $\S \S 4.1$ and 6). The improved correlations are expected, since we now compute optical richnesses with the same redshifts used for calculating the X-ray properties ( $L_{\mathrm{X}}$ and $T_{\mathrm{X}}$ taken directly from BAX). In $\S 4.1$ we detail the richness estimator, investigating its dependence on the cluster centroid, redshift, and aperture used. Then, in $\S 4.2 \mathrm{we}$ show the results - in terms of recovery rate - of the comparison between the BAX clusters and the NoSOCS. We begin by searching for possible optical counterparts in NoSOCS for each of the 792 BAX clusters, using a matching radius of $1.50 h^{-1} \mathrm{Mpc}$ and keeping only the closest match to each BAX entry. The total number of recovered systems is 638 .

\subsection{Systematic Effects on Richness Estimation}

Measurement of the optical richness of a cluster depends on the cluster centroid, radius adopted, and the redshift. Our richness measure $N_{\text {gals }}$ is defined as the number of galaxies at $m_{r}^{*}-1 \leq$ $m_{r} \leq m_{r}^{*}+2$ within a given aperture, where $m_{r}^{*}$ is the characteristic apparent magnitude of the cluster luminosity function. For this work we assume a universal Schecter luminosity function, with parameters given by Paolillo et al. (2001). The characteristic magnitude is $M_{r}^{*}=-22.17$, while $\alpha=-1.1$. As explained below, we find the optimal aperture to be $0.50 h^{-1} \mathrm{Mpc}$. We have considered no evolution in the characteristic magnitude $\left(m^{*}\right)$; $k$-corrections are applied to the magnitude of each galaxy, as

\footnotetext{
4 The NoSOCS catalog and other DPOSS products can be found at http:// dposs.ncsa.uiuc.edu/.
}

explained in steps 1 and 3 below. These corrections are obtained through the convolution of spectral energy distributions from Coleman et al. (1980) with the DPOSS $r$-filter. The steps taken to estimate richness are the following:

1. We use the redshift of each cluster to convert the characteristic magnitude to an apparent magnitude $\left(m_{r}^{*}\right)$ and to calculate the apparent radius (in arcseconds) for a fixed aperture of $0.50 h^{-1} \mathrm{Mpc}$. If a cluster lies closer than $0.50 h^{-1} \mathrm{Mpc}$ to a plate border we do not calculate its richness. The redshift is also used to compute the $k$-correction values typical of elliptical and late-type galaxies (Sbc) at the cluster redshift. These are named " $k e$ " and " $k s$," respectively. Because we only want to count galaxies with $m_{r}^{*}-1 \leq m_{r} \leq m_{r}^{*}+2$, we select all galaxies within $0.50 h^{-1} \mathrm{Mpc}$ of the cluster center at $m_{r}^{*}-1+k s \leq m_{r} \leq m_{r}^{*}+2+k e$. The $k$-corrections are applied to individual galaxies at a later stage, so these limits guarantee that we select all galaxies that can fall within $m_{r}^{*}-1 \leq m_{r} \leq m_{r}^{*}+2$. The number of galaxies selected in the cluster region is $N_{\text {clu. }}$. If the low $\left(m_{r}^{*}-1+k s\right)$ or high $\left(m_{r}^{*}+\right.$ $2+k e)$ magnitude limits fall outside the survey observational limits $\left(15.0 \leq m_{r} \leq 20.0\right)$, we apply a correction to the richness measure (explained below).

2. We estimate the background contribution locally. We randomly select 20 background boxes $\left(1200^{\prime \prime} \times 1200^{\prime \prime}\right)$ within an annulus ranging from $2.25 h^{-1} \mathrm{Mpc}$ to $2.25 h^{-1} \mathrm{Mpc}+1.3$ of the cluster center. Galaxies are selected within the same magnitude range as used for computing $N_{\text {clu }}$. We reselect boxes that overlap with an excised area of the survey (due to bright stars, for instance). The background counts of each box are scaled to the same area as the cluster, and the median counts (from all 20 boxes) gives the background estimate $\left(N_{\mathrm{bkg}}\right)$. We adopt the interquartile range (IQR, which is the range between the first and third quartiles) as a measure of the error in $N_{\mathrm{bkg}}$, which we term $Q_{\sigma_{\mathrm{bkg}}}$ (for normally distributed data $\mathrm{IQR}=1.35 \times \sigma$, where $\sigma$ is the standard deviation). The background-corrected cluster counts $\left(N_{\mathrm{clu}}-N_{\mathrm{bkg}}\right)$ is called $N_{\text {corr. }}$.

3. Next, a bootstrap procedure is used to statistically apply $k$-corrections to the galaxy populations in each cluster. In each of 100 iterations, we randomly select $N_{\text {corr }}$ galaxies from those falling in the cluster region $\left(N_{\text {clu }}\right)$. We then apply a $k$-correction to the magnitude of each galaxy. An elliptical $k$-correction is applied to $80 \%$ of the $N_{\text {corr }}$ galaxies, while an Sbc $k$-correction is applied to the remaining $20 \%$. Finally, we use these $k$-corrected magnitudes to count the number of galaxies at $m_{r}^{*}-1 \leq m_{r} \leq$ $m_{r}^{*}+2$. The final richness estimate $N_{\text {gals }}$ is given by the median counts from the 100 iterations. The richness error from the bootstrap procedure alone is given by $Q_{\sigma_{\text {boot }}}=\mathrm{IQR}$. The total richness error is the combination of this error and the background contribution, so that $Q_{\sigma_{N_{\text {gals }}}}=\left(Q_{\sigma_{\text {boot }}}^{2}+Q_{\sigma_{\mathrm{bkg}}}^{2}\right)^{1 / 2}$.

4. If the cluster is too nearby or too distant, either the bright $\left(m_{r}^{*}-1+k s\right)$ or faint $\left(m_{r}^{*}+2+k e\right)$ magnitude limit, respectively, will exceed one of the survey limits $\left(15.0 \leq m_{r} \leq 20.0\right)$. In this case we need to apply one of the following correction factors to the richness estimate:

$$
\begin{gathered}
\gamma_{1}=\frac{\int_{m_{r}^{*}-1}^{m_{r}^{*}+2} \Phi(m) d m}{\int_{15}^{m_{r}^{*}+2} \Phi(m) d m} \\
\gamma_{2}=\frac{\int_{m_{r}^{*}-1}^{m_{r}^{*}+2} \Phi(m) d m}{\int_{m_{r}^{*}-1}^{20} \Phi(m) d m}
\end{gathered}
$$

We call $\gamma_{1}$ and $\gamma_{2}$ the low- and high-magnitude limit correction factors. Whenever necessary, one of the above factors is multiplied 


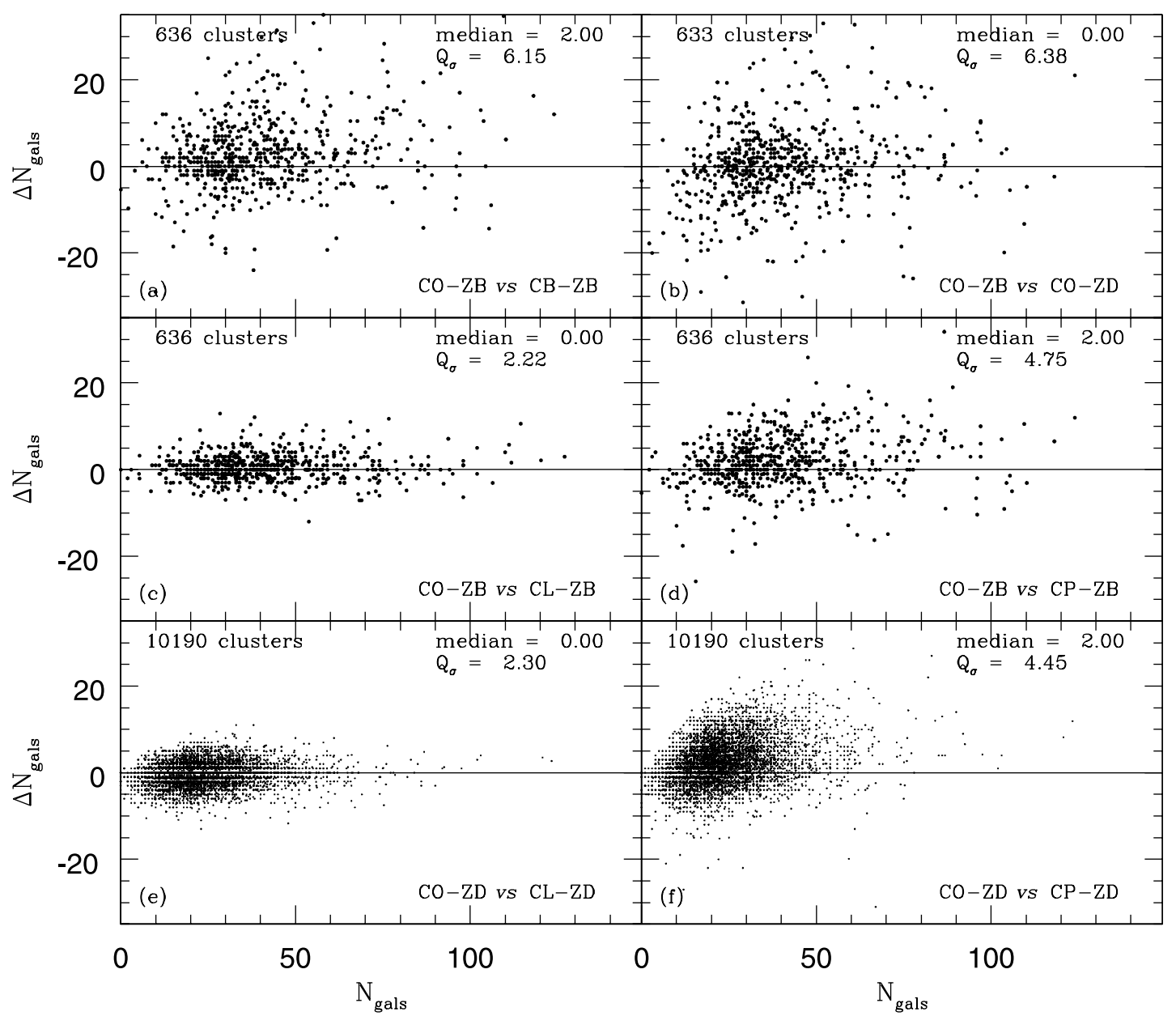

Fig. 1.-Richness residual for different cluster centroids and adopted redshifts. The labels in the bottom right of each figure have the following meaning: "C" stands for coordinates and "Z" for redshifts; "O" is the original cluster coordinate; "B" is the BAX centroid or redshift; " $\mathrm{L}$ " is the luminosity weighted coordinate; "P" is the centroid given by the maximum density peak; and "D" means the photometric redshift from DPOSS. Richness is compared for the following cases: (a) cluster original center vs. BAX centroid, both using the BAX redshift; $(b)$ using the DPOSS redshift vs. the BAX redshift (both at the original coordinates); $(c)$ original center vs. the luminosity weighted coordinate (both using the BAX redshift); $(d)$ original center vs. the density peak centroid (both using the BAX redshift); $(e)$ same as $(c)$, but for the 10,190 clusters used to estimate substructure; $(f)$ same as $(d)$, but for all 10190 clusters in the NoSOCS sample. Panels $e$ and $f$ use the DPOSS photometric redshift by necessity.

by $N_{\text {gals. }}$ Because the optical data span 5 mag it is impossible to simultaneously exceed both the bright and faint end.

An estimate of the cluster contrast is also obtained along with the richness. The contrast is defined as the ratio between the number of galaxies in the cluster region having $m_{r}^{*}-1+k s \leq m_{r} \leq$ $m_{r}^{*}+2+k e\left(N_{\mathrm{clu}}\right)$, and the background error $Q_{\sigma_{\mathrm{bkg}}}$ (estimated within the same magnitude range).

We utilize this richness measure to examine its dependence on the cluster centroid, radius, and redshift. In Figure 1 we show the residual between different richness estimates obtained when adopting different centroids and redshifts. In the bottom right corner of each panel, we encode the coordinates " $C$ " and redshifts " $Z$ " used as follows:

1. $\mathrm{CO}$, the original cluster position from NoSOCS, which is the location provided by SExtractor in an adaptively smoothed density map.

2. CL, a luminosity weighted coordinate, where each galaxy position within the cluster aperture is weighted by the galaxy's luminosity and a new center is computed.

3. $\mathrm{CB}$, the cluster center cataloged in BAX.

4. $\mathrm{CP}$, the coordinate of the maximum density peak.

5. ZD, the photometric redshift from the DPOSS data.

6. ZB, the redshift (usually but not always spectroscopic) cataloged in BAX.
In the two bottom panels we show the results obtained with all 10190 clusters used to estimate substructure $(\S 5)$, while in the top four panels the analysis is restricted to the 636 clusters common to BAX and DPOSS.

From inspection of this figure it is clear that the richnesses estimated with the X-ray centroids are underestimated compared to those obtained with the optical centers (Fig. 1a). This is likely due to the optical center being a better choice than the X-ray center to trace the distribution of galaxies within the cluster. If we restrict the study to clusters with small optical-X-ray centroid offsets $\left(\lesssim 0.50 h^{-1} \mathrm{Mpc}\right)$ the results are very similar, although a small trend for higher richness values computed with the optical centers is expected. When adopting the same center (optical) and using different redshifts (from BAX and DPOSS) there is no obvious trend (the median residual is zero), but the scatter is the largest of all the comparisons (Fig. 1b). This implies that there is no systematic difference between the photometric redshifts and those from BAX. The scatter arises from a shift in sampling the luminosity function of each cluster and a change in radius when estimating richness. Adopting either the original or the luminosity weighted coordinates does not appreciably affect the resultant richnesses (Figs. $1 c$ and $1 e$ ), as the two centers are typically similar. On the other hand, the richness estimate obtained with the density peak center is underestimated in comparison to the 

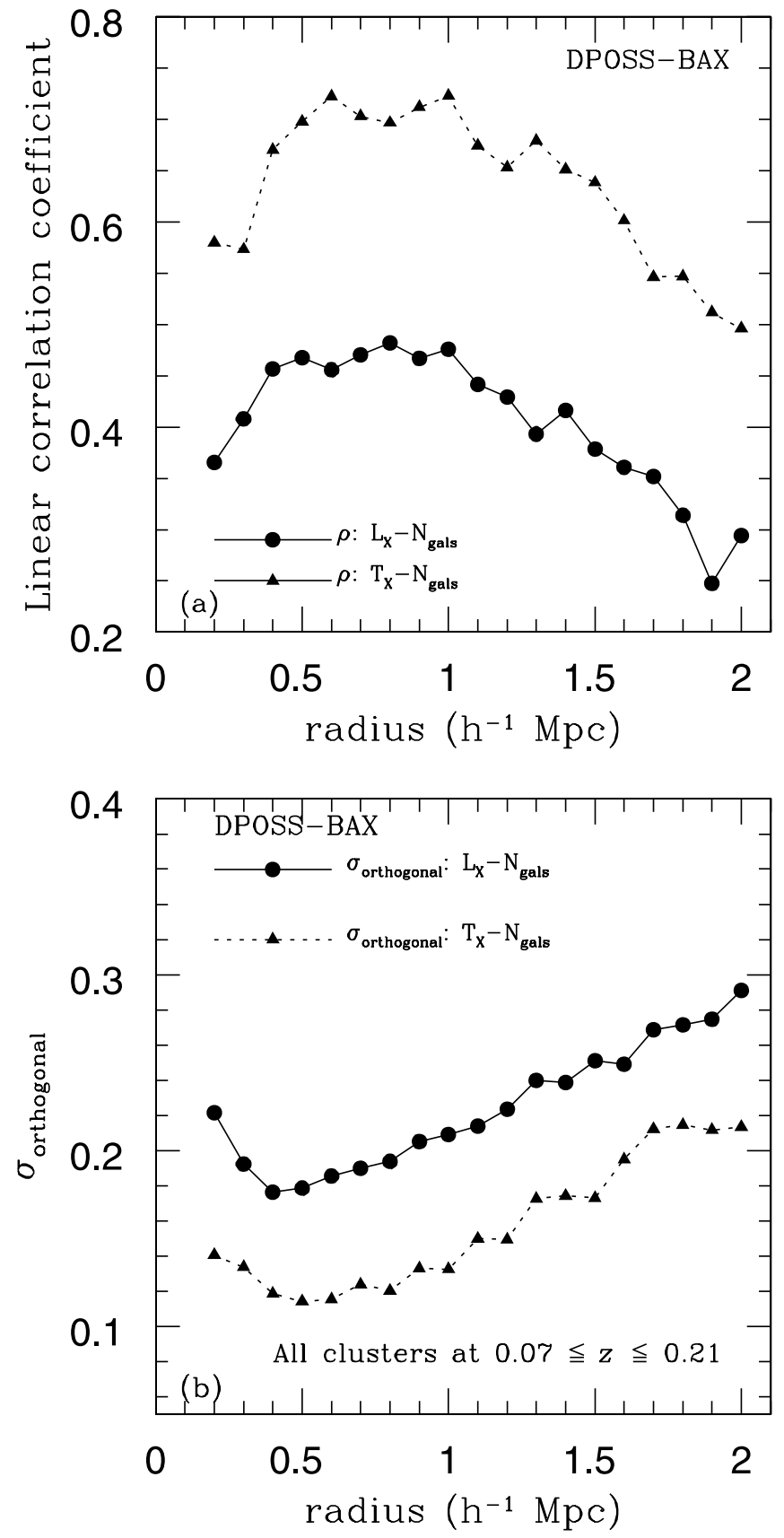

FIG. 2.-Top: Dependence of the linear correlation coefficient $(\rho)$ for the $L_{\mathrm{X}}$ $N_{\text {gals }}$ (solid line) and $T_{\mathrm{X}}-N_{\text {gals }}$ relations (dotted line) as a function of the radius; Bottom: Variation of the orthogonal scatter of the $L_{\mathrm{X}}-N_{\text {gals }}$ (solid line) and $T_{\mathrm{X}}-N_{\text {gals }}$ relations (dotted line) as a function of the radius used for calculating richness.

other optical centroids (Figs. $1 d$ and $1 f$ ). This may be due to the presence of substructure (or projection effects), as a cluster showing more than one galaxy concentration within the measurement aperture $\left(0.50 h^{-1} \mathrm{Mpc}\right)$ will have multiple density peaks. Choosing the highest density peak for richness calculation-i.e., counting galaxies within $0.50 h^{-1} \mathrm{Mpc}$ from this new center - may not be appropriate for tracing the galaxy distribution. If the cluster is defined by a single peak, then the richness estimate should not be affected.

Beyond considering the effects mentioned above, we investigate which metric aperture is optimal for computing the richness. In Gal et al. (2003) we adopted a $1.00 h^{-1}$ Mpc radius, which may not be the best choice for the current purposes. Like Popesso

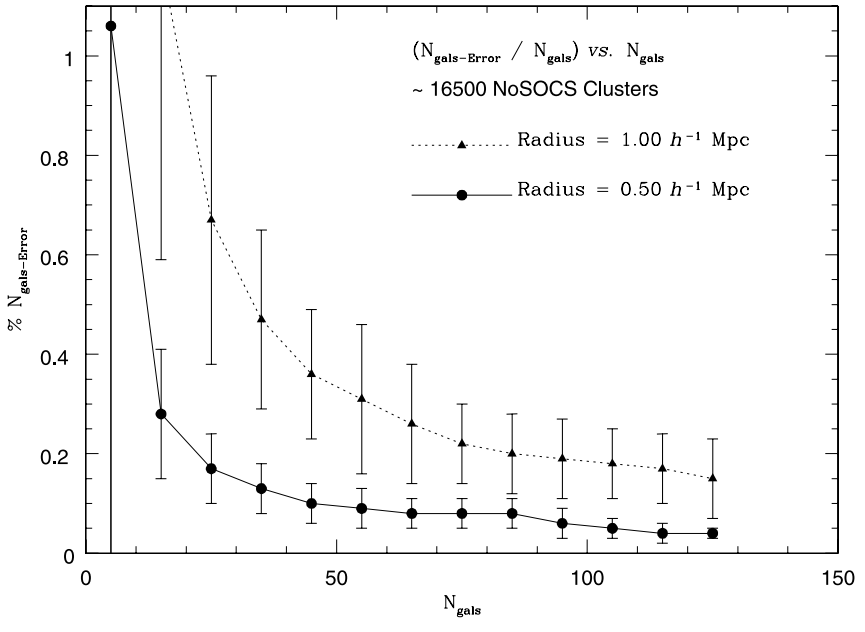

FIG. 3.-Relative richness error as a function of richness for the full NoSOCS sample ( $\sim 16,500$ clusters). The solid line is the variation of relative richness error using a $0.50 \mathrm{~h}^{-1} \mathrm{Mpc}$ aperture for computing richness. The variation when adopting a radius of $1.00 \mathrm{~h}^{-1} \mathrm{Mpc}$ is shown by the dotted line.

et al. (2004), we used the scaling relations between optical and $\mathrm{X}$-ray properties to guide the choice of the optimal radius. We investigated how the scatter of these relations is affected by the different radii used for computing richness, examining the $L_{X^{-}}$ $N_{\text {gals }}$ and $T_{\mathrm{X}}-N_{\text {gals }}$ relations based on all clusters common to BAX and DPOSS used in the substructure analysis (see $\S 5$ ). Figure 2 shows the variation of the linear correlation coefficient $(\rho)$ and the orthogonal scatter $(\sigma)$ for these two relations with the aperture used to estimate richness. We see a clear trend for minimum scatter and highest correlation for an aperture of $\sim 0.50 h^{-1} \mathrm{Mpc}$. In Figure 3 we show the relation between the relative richness error $\left(N_{\text {gals-err }} / N_{\text {gals }}\right)$ and richness for two apertures. We see that clusters with $N_{\text {gals }}<10$ (within $0.50 h^{-1} \mathrm{Mpc}$ ) have errors comparable to their richnesses. The increase in the richness error as we increase the radius from 0.50 to $1.00 h^{-1} \mathrm{Mpc}$ is also striking. Based on these three plots and on Table 1 (where we show the scatter of the optical versus X-ray relations for richness estimates based on different centroids and redshifts), we recompute the richness estimate ( $\left.N_{\text {gals }}\right)$, instead of using the values from Papers II and III. We adopt the original optical cluster position, the BAX redshift and an aperture of $0.50 h^{-1} \mathrm{Mpc}$ for each cluster, and use the BAX cosmology. We also exclude clusters with $N_{\text {gals }}<10$ (unless otherwise stated) for the comparisons shown in $\S 6$. This step (recomputing richness) is crucial for minimizing the scatter in the comparisons between optical and X-ray properties ( $\S 6$ and Table 1). The impact of centroid, radius and redshift on substructure measurement is discussed in $\S 5$.

TABLE 1

Scatter and Number of Objects in the Optical versus X-Ray Relations for Different Richness Estimates

\begin{tabular}{|c|c|c|c|c|}
\hline \multirow[b]{2}{*}{ SET } & \multicolumn{2}{|c|}{$L_{\mathrm{X}}-N_{\mathrm{gals}}$} & \multicolumn{2}{|c|}{$T_{\mathrm{X}}-N_{\text {gals }}$} \\
\hline & All Clusters & $\beta_{\text {signif }>0.05}$ & All clusters & $\beta_{\text {signif }>0.05}$ \\
\hline CO-ZB................... & $0.172 ; 430$ & $0.171 ; 271$ & $0.112 ; 53$ & $0.041 ; 23$ \\
\hline CB-ZB $\ldots \ldots \ldots \ldots \ldots$ & $0.184 ; 418$ & $0.174 ; 233$ & $0.119 ; 53$ & $0.061 ; 26$ \\
\hline CO-ZD ………........ & $0.179 ; 424$ & $0.175 ; 266$ & $0.111 ; 48$ & $0.111 ; 29$ \\
\hline CL-ZB ………..... & $0.174 ; 432$ & $0.174 ; 274$ & $0.120 ; 53$ & $0.062 ; 28$ \\
\hline CP-ZB ………..... & $0.174 ; 426$ & $0.175 ; 260$ & $0.120 ; 53$ & $0.065 ; 26$ \\
\hline
\end{tabular}




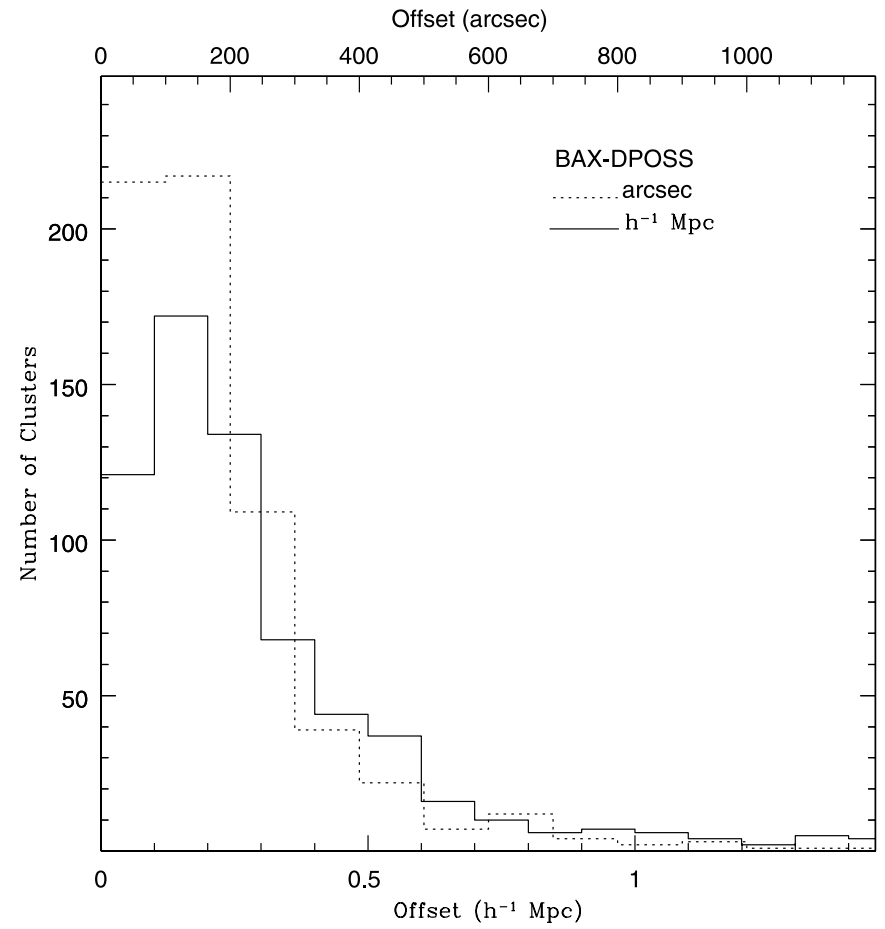

FIG. 4.-Offset distribution between BAX and NoSOCS clusters in $h^{-1} \mathrm{Mpc}$ (solid line) and arcseconds (dotted line).

\subsection{Comparison of the Two Catalogs}

We compare the BAX and NoSOCS catalogs using BAX as the reference, searching for optical counterparts in NoSOCS. We adopt a search radius of $1.50 \mathrm{~h}^{-1} \mathrm{Mpc}$, keeping only the nearest match to each of the 792 BAX clusters. The total number of recovered systems is 638 . In Figure 4 we show the offset distribution in $h^{-1} \mathrm{Mpc}$ (solid line) and arcseconds (dotted line). The typical X-ray-optical offset is $<0.50 h^{-1} \mathrm{Mpc}$ (or $<400^{\prime \prime}$ ). The redshift and richness distributions of the common and missing clusters are shown in Figure 5, as the top left and right panels, respectively. The majority of BAX clusters not identified by NoSOCS are at higher redshifts $(z \gtrsim 0.2)$ and have low-richness $\left(N_{\text {gals }}<40\right)$ systems. For clusters not found by NoSOCS, we adopt the X-ray coordinates (given in BAX) to compute their richnesses.

The overall recovery rate of BAX clusters by NoSOCS is $81 \%$. Considering the redshift and richness (or $L_{\mathrm{X}}$ ) ranges sampled this is an encouraging result. It is also important to keep in mind that the BAX catalog is a compilation of all X-ray galaxy clusters found in the literature and not a complete list to a given flux limit. Instead of trying to explain each missing cluster on a case-by-case basis, we investigate their overall properties (richness and $\left.L_{\mathrm{X}}\right)$ as a function of redshift. This approach also enables us to directly compare our results to the selection functions (SF) estimated by Gal et al. (Papers II and III). The recovery rate of X-ray-emitting clusters by NoSOCS is shown in the bottom panels of Figure 5. In the left panel we show the detection rate for different richness classes, while in the right panel the relation is shown for different X-ray luminosities. Comparison of the left panel of this figure to Figure 6 from Paper II suggests that the earlier simulations performed to evaluate the SFs tend to underestimate the successful detection rate. However, the BAX list used here is not a complete catalog and includes clusters that were first found in the optical and only later observed at X-ray wavelengths. Even considering these two points the results shown in the bottom panels of Figure 5 are quite impressive, demonstrating a recovery rate of approximately $90 \%$ of all X-ray luminous $\left(L_{X} \gtrsim 3.210^{44} \mathrm{ergs}^{-1}\right)$ galaxy clusters at any redshift out to $z \sim 0.2$. In terms of richness, we recover all X-ray clusters cataloged in BAX that have $N_{\text {gals }} \gtrsim 80$ out to $z \sim 0.3$, and more than $90 \%$ of those with $N_{\text {gals }} \gtrsim 25$ to $z \sim 0.2$.

\section{OPTICAL SUBSTRUCTURE}

We have used photometric data alone to obtain an estimate of the fraction of clusters with evidence for substructure, based on our sample of 10,190 clusters at $0.07 \lesssim z \lesssim 0.21$. In order to evaluate substructure, we apply four two-dimensional (spatial) statistical tests (Pinkney et al. 1996). It is well known that any substructure test statistic has little meaning if not properly normalized, which can be achieved by comparing the results for the input data to those for substructure-free samples (the "null hypothesis"). For the two-dimensional tests employed in this work the null hypothesis is given by an azimuthally symmetric, smooth distribution of points, with surface density decreasing as a function of radius. We generate such data via azimuthal randomization, where the distance of each galaxy to the cluster center is maintained and a new azimuth is randomly assigned. The main advantage of this technique is the exact replication of the radial profile of the cluster. The four tests used here each provide a significance level, given as the probability that the observed distribution is drawn randomly from one free of substructure.

The significance level is determined through Monte Carlo simulations. For each input data file we generate $N=500$ simulated data sets by azimuthal randomization. We then calculate the number of Monte Carlo simulations which show more substructure than the real data. Finally, this number is divided by the number of Monte Carlo simulations. For most of the analysis in this paper we set our significance threshold at 5\%, meaning that only 25 of the 500 simulated data sets can have substructure statistics higher than the observations to consider a substructure estimate significant. This choice of threshold is explained in $\S 5.5$.

In the next four subsections we give a brief description of each substructure estimator: the "angular separation test" (AST), the "Fourier elongation test" (FE), the "Lee statistic" (Lee 2D), and the "symmetry test" $(\beta)$. Detailed descriptions of all four tests are provided by Pinkney et al. (1996).

\subsection{The Angular Separation Test}

The angular separation test was developed by West et al. (1988) and considers the galaxy distribution in the cluster only in terms of angular coordinates around the cluster center. In this case, the presence of substructure is detected as an excess number of small angular separations between galaxy pairs relative to the expected number in a spherically symmetric, substructure-free distribution. The harmonic mean angular separation is given by

$$
\theta_{\mathrm{hm}}=\left[2 / N(N-1) \sum_{i>j} \theta_{i j}^{-1}\right]^{-1}
$$

where the sum is performed over all pairs; $N$ is the total number of galaxies, and $\theta_{i j}$ is the angular separation between galaxies $i$ and $j$. The test statistic is the ratio of $\theta_{\mathrm{hm}}$ measured for the cluster and the harmonic mean for a Poisson distribution with the same number of galaxies, AST $=\theta_{\mathrm{hm}} / \theta_{P}$. This ratio is near unity for substructure-free systems, and less than 1.0 for clumpy distributions; $\theta_{P}$ is obtained from Monte Carlo simulations whose sets are generated using the azimuthal randomization described above. The test also adopts a small angle filter, rejecting all $\theta_{i j}$ 

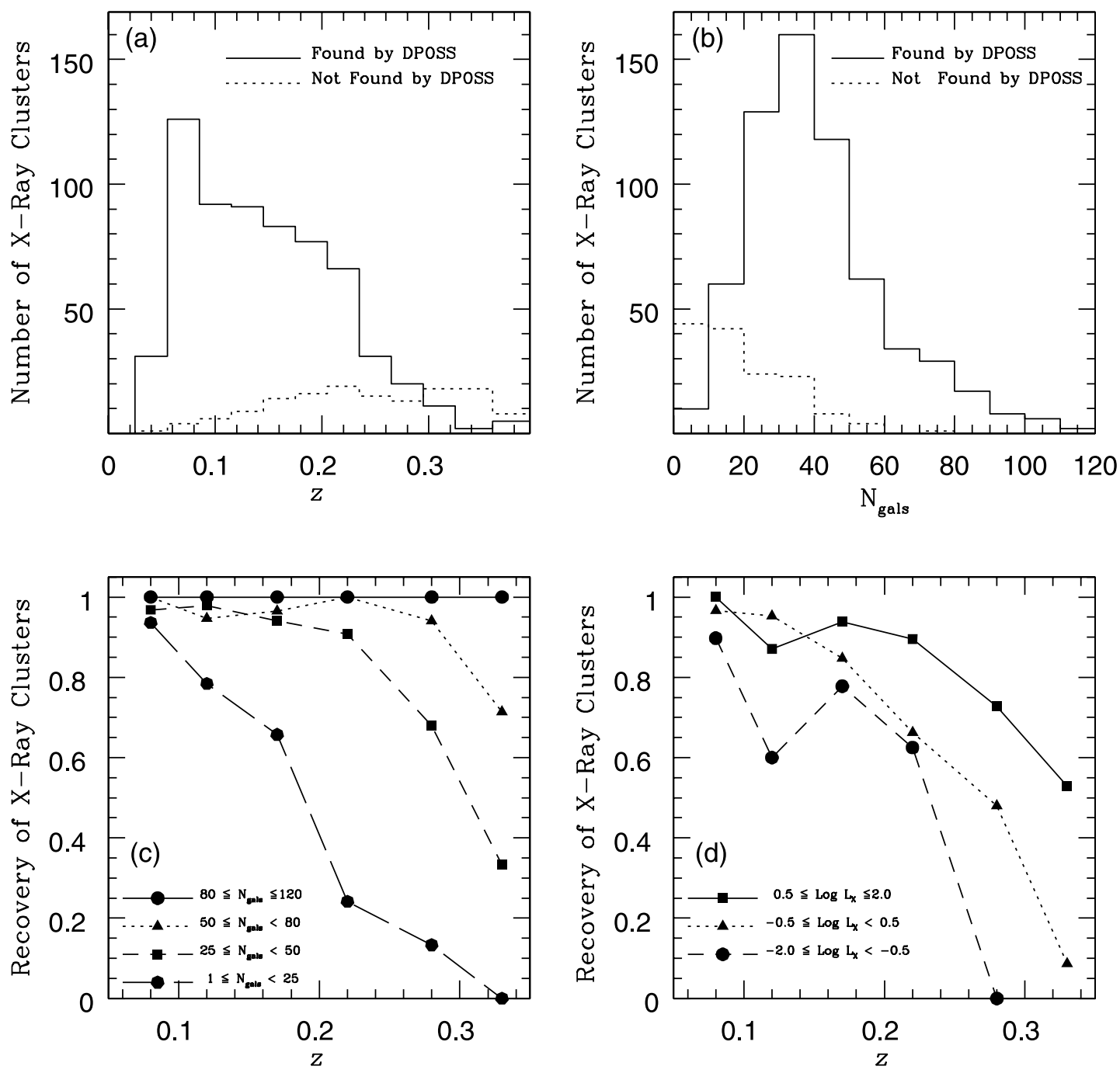

FIG. 5.-Recovery rate of BAX clusters in NoSOCS: (a) redshift distribution for common clusters and BAX clusters not found in DPOSS; $(b)$ the richness distributions for common objects and BAX only sources; $(c)$ recovery rate of X-ray clusters by NoSOCS, as a function of redshift, for different richness classes $\left(1 \leq N_{\text {gals }}<25\right.$; $\left.25 \leq N_{\text {gals }}<50 ; 50 \leq N_{\text {gals }}<80 ; 80 \leq N_{\text {gals }} \leq 120\right)$; and $(d)$ the same as in $(c)$, but for different X-ray luminosity ranges $\left(-2.0 \leq 1 \log L_{\mathrm{X}}<-0.5 ;-0.5 \leq\right.$ $\left.\log \bar{L}_{\mathrm{X}}<0.5 ; 0.5 \leq \log L_{\mathrm{X}} \leq 2.0\right)$.

that are less than $1 \%$ of the expected mean intergalaxy separation if the $N$ galaxies were uniformly distributed $(0.01[2 \pi / N])$. West et al. (1988) find that this test is too sensitive to the shape of the cluster density profile to provide unambiguous evidence for the presence of substructure. The main disadvantage of this test is the loss of information when going from two spatial dimensions to a single angular dimension. However, as pointed out by Pinkney et al. (1996), AST may be a more sensitive diagnostic for clumping in multiple systems.

\subsection{The Symmetry Test}

The $\beta$ (or symmetry) test was also introduced by West et al. (1988) to test for significant deviations from mirror symmetry about the cluster center. This test assumes that a subcluster represents a local asymmetry superposed on an otherwise symmetric distribution. For each galaxy $i$, a local density estimate is obtained by the mean distance to the $N^{1 / 2}$ nearest neighbors $d_{i}$ (West et al. 1988 used the five nearest neighbors). The local density for a point $o$ diametrically opposite to galaxy $i$ is estimated in the same way (estimating the mean distance to the $N^{1 / 2}$ nearest galaxies, $d_{o}$ ). For a symmetric galaxy distribution the values of $d_{i}$ and $d_{o}$ should, on average, be approximately equal, but they will differ for clumpy distributions. The asymmetry for a given galaxy $i$ is given by

$$
\beta_{i}=\log \left(\frac{d_{o}}{d_{i}}\right)
$$

The $\beta$-statistic is then defined by the average value $\left\langle\beta_{i}\right\rangle$ over all galaxies. For a symmetric distribution $\langle\beta\rangle \approx 0$, while values of $\langle\beta\rangle$ greater than 0 indicate asymmetries. $\langle\beta\rangle$ is a densityweighted average, being more sensitive to the presence of substructure than if random points were selected from the cluster. The $\beta$-test is sensitive to deviations from mirror symmetry, but not necessarily to ones from circular symmetry. In other words, the test does not mistake an elongated smooth cluster for one containing substructure.

\subsection{The Fourier Elongation Test}

One of the characteristics of galaxy clusters is elongation, which can be taken as a substructure indicator (although not a definitive one). In most cases, clusters with elongated galaxy distributions will have substructure. We use Fourier analysis (Rhee et al. 1991a) to estimate the elongation of galaxy clusters. In this 
method we assume the azimuthal galaxy distribution $[N(\phi)]$ resembles the model

$$
N(\phi)=\left(\frac{N_{0}}{2 \pi}\right)\left[1+\left(N_{1} / N_{0}\right) \cos \left(2 \phi-2 \phi_{0}\right)\right],
$$

where $N_{0}$ is the number of cluster galaxies, $\phi_{0}$ is the cluster position angle and $N_{1}$ is the elongation amplitude. The statistic for this test is given by the elongation strength, defined by

$$
\mathrm{FE}=\frac{N_{1}}{\sqrt{2 N_{0}}}=\frac{2\left(S^{2}+C^{2}\right)^{1 / 2}}{\sqrt{2 N_{0}}},
$$

where

$$
S=\sum N(\phi) \sin (2 \phi), \quad C=\sum N(\phi) \cos (2 \phi) .
$$

Pinkney et al. (1996) recommend a more strict criteria when using FE to detect substructure. They advise using FE to reject the circular hypothesis at the $1 \%$ level, with FE being greater than 2.5. This is motivated by the fact that elongation is also a signature of other formation scenarios besides hierarchical mergers. Rather than adopt these somewhat arbitrary criteria, we optimize this test the same way as the others $(\S 5.5)$. The first criterion $(1 \%$ rejection level) may be too rigorous, while the second (selecting only clusters with FE $>2.5$ ) makes little difference to the final results (Fig. 9).

\subsection{The Lee Statistic}

The Lee statistic (Lee 1979) tests for bimodality in a given distribution, which is perhaps the simplest type of substructure. In our case, we test the hypothesis that the galaxies in a cluster lie in two clumps. Since its introduction in 1979, the test has been used in astronomical applications, e.g., Fitchett \& Webster (1987) and Rhee et al. (1991b). The algorithm works as follows: (1) The data are projected onto a line, which makes an angle $\phi$ with a second line, assumed to be of constant declination. (2) The first line is then rotated by small increments for $0^{\circ} \leq \phi \leq 180^{\circ}$. (3) For each orientation all points are projected onto the second line, so that each point assumes a new coordinate, $x_{j}$, along that line. (4) A search for the best partition into two clumps (named "left" and "right") is performed. The quantities $\sigma_{l}, \sigma_{r}$, and $\sigma_{T}$ are calculated for the left, right, and total samples, respectively, for the $N-1$ partitions. The $\sigma$ are given by $\sigma=\sum\left(x_{j}-\mu\right)^{2}$, with $\mu$ given by $\sum x_{j} / n$. The quantity $L$ is then defined by

$$
L=\max _{\text {partitions }}\left(\frac{\sigma_{T}}{\sigma_{l}+\sigma_{r}}-1\right) .
$$

$L$ varies with $\phi$, becoming large when the projection axis connects two distinct clumps in the original data set. Following Fitchett (1988), we adopt $L_{\text {rat }}=L_{\max } / L_{\min }=\max L(\phi) / \min L(\phi)$ as this test's statistic. As in Pinkney et al. (1996) we use $6^{\circ}$ increments in $\phi$. As pointed out by Pinkney et al. (1996), the Lee $2 \mathrm{D}$ test is a conservative test because it does not detect elongations as substructure and also loses sensitivity in the presence of more than two subclusters (or if there are two clumps, but their sizes are dissimilar).

\subsection{Summary of Substructure Tests}

The codes used for the substructure analysis are those of Pinkney et al. (1996), who evaluated the performance of 31 statistical tests (including the four two-dimensional tests described above) on $N$-body simulations of galaxy cluster mergers. For the twodimensional tests they recommend three diagnostics for substructure studies: FE, Lee 2D, and $\beta$. Based on the simulations, they find that the $\beta$-test is the most sensitive, while the AST is very insensitive as it requires a distinct compact subcluster to detect substructure. The FE test is very sensitive to elongations, but they advise setting a threshold for the FE statistic. The Lee $2 \mathrm{D}$ test requires the presence of a very distinct subcluster, and is insensitive to more than two clumps (or two clumps with very different sizes). Pinkney et al. (1996) point out that Lee 2D is sensitive only to genuine two-dimensional substructure, while $\mathrm{FE}$ and $\beta$ are able to find substructure in a cluster that is not necessarily a merger. These conclusions are based on their extensive simulations, and we do not have sufficiently detailed data to independently test their assertions. We describe below how we optimize the tests and the results when they are applied to the DPOSS data.

Pinkney et al. (1996) conclude with a note saying that, when galaxies have no redshifts, substructure could be erroneously detected due to the superposition of foreground and background groups. While this is certainly true, the large amount of twodimensional data available from the most recent sky surveys will suppress this effect. The acquisition of deep X-ray data or galaxy velocities for a large sample of moderately redshift galaxy clusters is very expensive, observationally speaking. Thus two-dimensional data are also useful for the selection of subsamples for future surveys (Kriessler \& Beers 1997). Kolokotronis et al. (2001) used an alternate approach when computing the fraction of optical substructure estimates that are due to background contamination, searching for substructure using two-dimensional optical and X-ray imaging data for 22 clusters. They find good agreement between optical and X-ray results, with only $\sim 22 \%$ of the clusters yielding inconsistent results (namely, substructure seen in the optical, but not in the X-ray). They argue that this difference is probably due to projection effects in the optical data. However, it is important to point out that some recent studies have found that projection can also affect the morphology inferred from X-ray data (López-Cruz et al. 2004; Rines \& Diaferio 2006). In our case, we try to minimize this problem by using only galaxies selected in a given magnitude range (an issue that is not very well addressed in previous works). We have also optimized our methods to minimize the scatter when comparing optical and X-ray properties (e.g., $T_{\mathrm{X}}-N_{\text {gals }}$, described below).

\subsection{Dependence on Input Data}

The substructure results we show below are based on 10190 NoSOCS clusters at $0.07 \lesssim z \lesssim 0.21$, which is the largest sample used for this type of study to date. The rationale for using only clusters at these redshifts is detailed below. For the optimization of the tests we have used the subsample of 445 clusters with a counterpart in BAX. We first need to quantify the sensitivity of the cited tests to the choices of centroid, redshift, magnitude range, maximum radius, and significance level by evaluating substructure for different ranges of these parameters. In Figure 6 we show the effects of the adopted center and redshift. We test four centers, the original cluster position $(\mathrm{O})$, the BAX coordinate $(\mathrm{B})$, the luminosity-weighted center $(\mathrm{L})$, and the position of the maximum density within the cluster $(\mathrm{P})$. The six panels in this figure are analogous to those in Figure 1, now showing the residuals in the $\beta$-test. In addition, we show in the top right of each panel the value of IQR for the residuals of the significance of the $\beta$-test (not plotted; these are labeled $Q_{\sigma 2}$ ). The largest differences are found when comparing the original cluster position to the BAX coordinate (or the density peak position) and when adopting different 


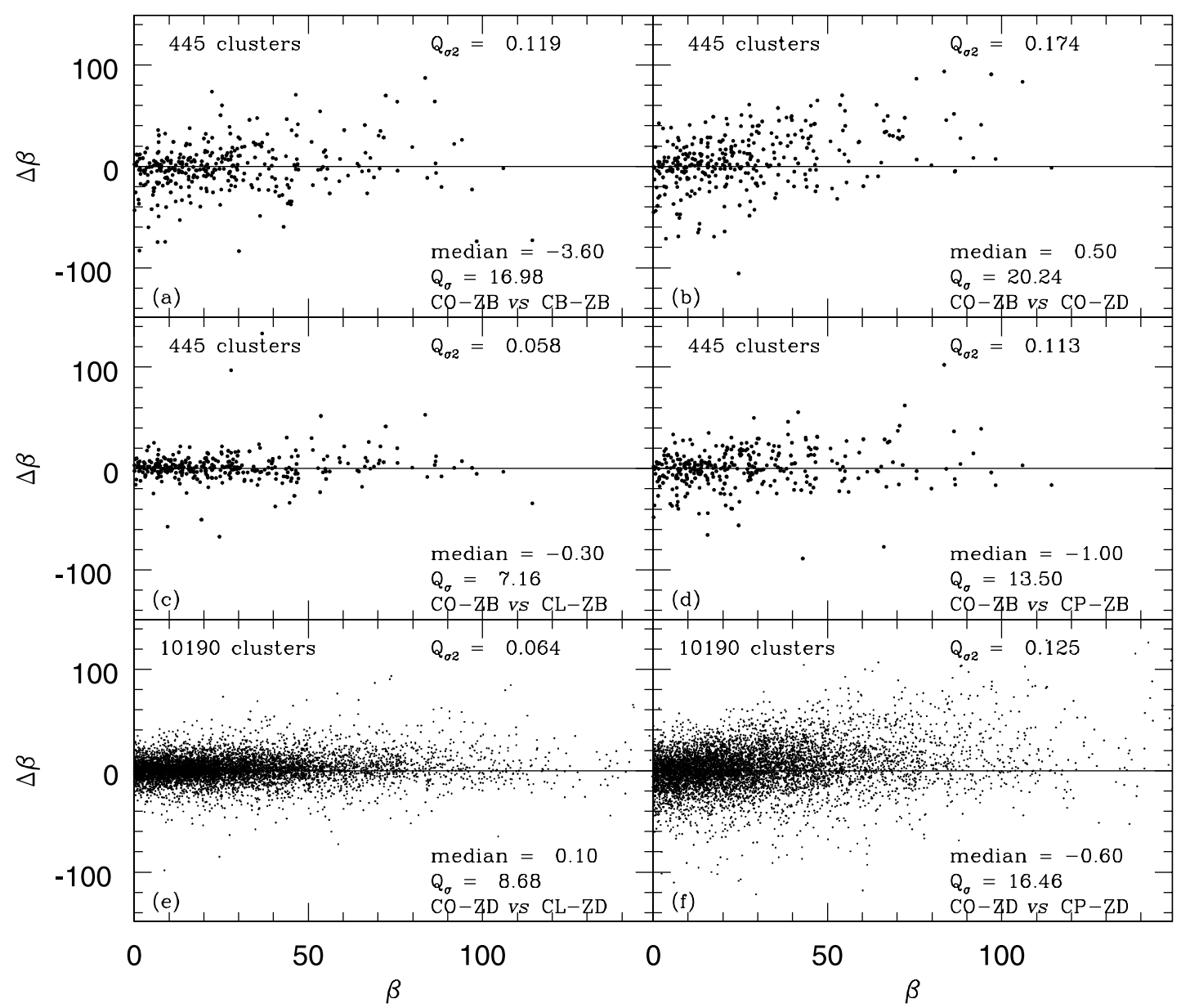

FIG. 6. - The $\beta$-test residual for different cluster centroids and adopted redshifts. The comparison shown in each panel is analogous to those in Fig. 1. The IQR value of the $\beta$-test residual is labeled $Q_{\sigma}$. In addition, we show in the top right of each panel the values of IQR (named $Q_{\sigma 2}$ ) for the residuals of the significance of the $\beta$-test.

redshifts (BAX or DPOSS). The centroid choice affects the general galaxy distribution, which may not be well indicated by the Xray center or maximum density peak (see the comments regarding Fig. 1). The redshift affects both the sampling of the cluster luminosity function and the apparent radius used for selecting galaxies for the substructure tests.

Based on this figure and on Table 1 we evaluate substructure (for the entire sample of 10,190 clusters) using the original cluster position, instead of the luminosity-weighted center or the density peak coordinate. For consistency, when using substructure to exclude clusters in comparisons of optical and X-ray properties (§ 6) we also adopt the original cluster position and the BAX redshifts (see the richness discussion). As shown in Table 1 this gives the minimum scatter for the optical-X-ray relations based on the substructure results.

In Figure 7 we show the dependence of the substructure results on the magnitude range (bottom panel) and maximum radius (middle panel) used when selecting projected cluster galaxies. Due to the large computational cost of running these tests for the entire sample of NoSOCS clusters, we show the dependence on the magnitude range and maximum radius only for the subset common to BAX. Obviously, the ability to detect substructure decreases when considering only the bright central galaxies. Conversely, at fainter flux limits and larger radii, the two-dimensional data presents problems due to background and foreground contamination. We first attempted to estimate substructure using all galaxies within the survey magnitude limits $(15.0 \leq r \leq 19.5)$, but this leads to misleading results due to different sampling of cluster galaxies for clusters at different redshifts. We thus investigated the dependence of the number of clusters with substructure on the magnitude range used. We tested six ranges, as indicated in the bottom panel of Figure 7, using only 100 (out of 638) clusters that span the full magnitude range sampled $\left(m_{r}^{*}-2 \leq\right.$ $m_{r} \leq m_{r}^{*}+2$, corresponding to redshifts of $0.11 \lesssim z \lesssim 0.14$ ).

We conclude that the high percentage of clusters with substructure when using faint galaxies $\left(m_{r}^{*}+2\right)$ may be an artifact due to background contamination. We do not require the brightest galaxies $\left(m_{r}^{*}-2\right)$ because that would further reduce our sample, eliminating lower redshift clusters. As shown in Figure 7, the results for the ranges $m_{r}^{*}-2 \leq m_{r} \leq m_{r}^{*}+1$ or $m_{r}^{*}-1 \leq$ $m_{r} \leq m_{r}^{*}+1$ are similar, having little effect on our conclusions. For all substructure analyses we therefore use the latter range, which is fully sampled by our data at redshifts $0.07 \lesssim z \lesssim 0.21$, where we find 445 of the 638 common BAX and NoSOCS clusters ( 430 of the 618 with estimates of $L_{\mathrm{X}}$ and $N_{\text {gals }}$ ) and 53 of 101 with estimates of $T_{\mathrm{X}}$. From the 16,546 clusters in NoSOCS there are 10,190 in this redshift range.

From the middle panel of Figure 7 we see that out to $R=$ $1.00 h^{-1} \mathrm{Mpc}$ the substructure tests behave similarly, except for AST, which is recognized by Pinkney et al. (1996) as being very insensitive. In the top panel of Figure 7 we show the dependence of the number of clusters with substructure on the significance level (S.L.) required to reject the null hypothesis. Obviously, as we relax the threshold to consider a substructure estimate meaningful, the number of selected clusters increases. Our choice of significance level is explained below. 

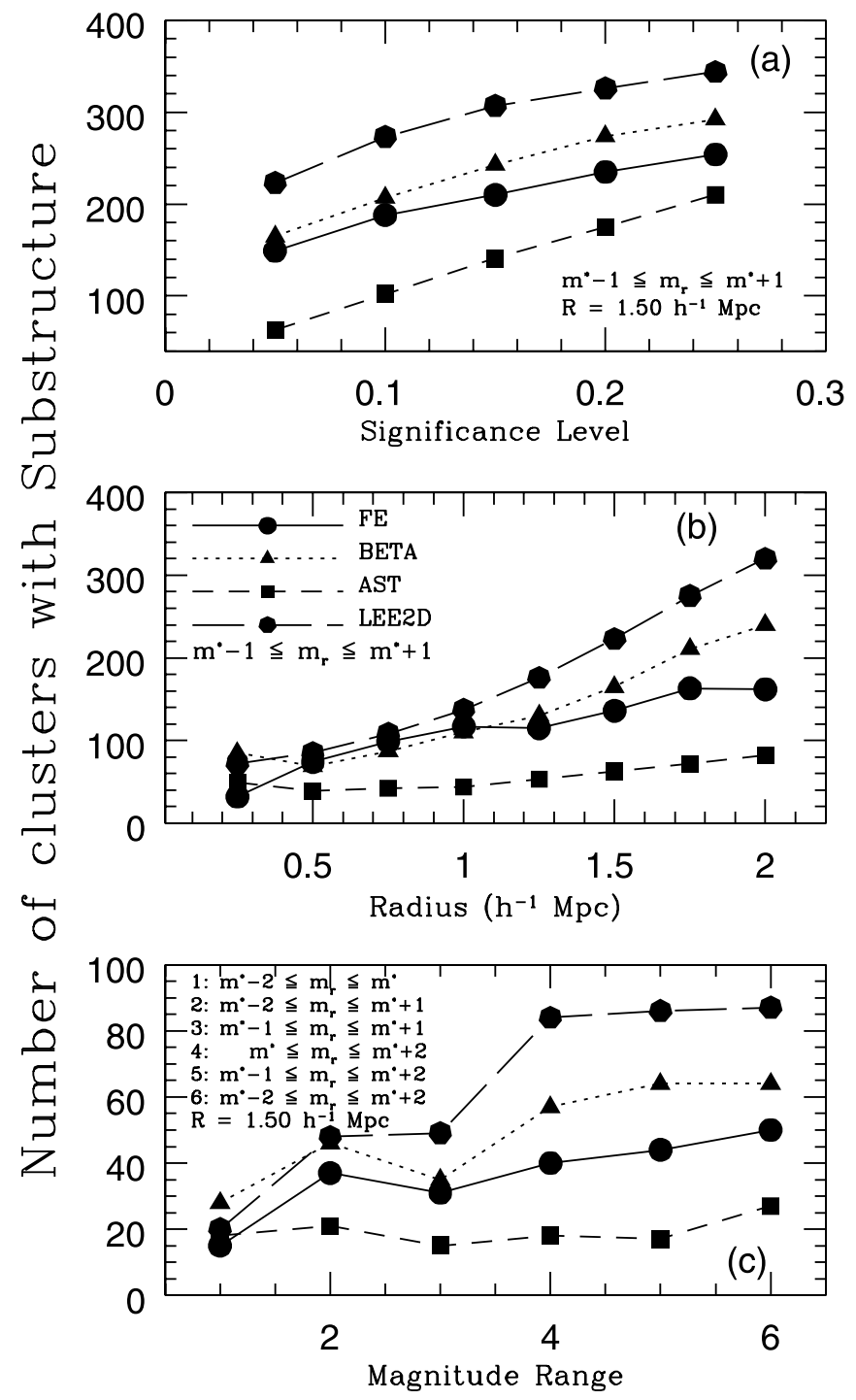

FIG. 7.- Substructure results for the four statistical tests as a function of the magnitude range (identified by an index from 1 to 6) sampled for each cluster (bottom panel), the radius (middle panel), and the significance level required for each test (top panel).

Any optical study relying on purely projected galaxy positions is affected by superpositions of loose groups. One could assess projection effects in substructure estimates by using X-ray data (Kolokotronis et al. 2001) or including velocity dispersion information. Given the large number of clusters in our sample, this is not practical. Since our goal is to compare optical and X-ray cluster properties $(\S 6)$ we optimize the parameters (magnitude range, radius, and S.L.) for the substructure tests by minimizing the scatter in these scaling relations. This could, in principle, lead to similar results as those obtained by Kolokotronis et al. (2001). This strategy is also complementary to the analysis in Figure 7, which gives no objective criteria to choose the optimal parameters.

This approach is similar to the one show in Figure 2. Before, we wanted to find the optimal radius to compute richness (the one that minimizes the scatter of the scaling relations). Now we want to find the optimal radius, magnitude range and S.L. to estimate substructure. Note that the optimal radius for substructure measurement need not be equal to the one used for richness, as we are measuring different cluster properties.

In the top panels of Figure 8 we examine how the orthogonal scatter of three correlations of cluster parameters $\left(L_{\mathrm{X}}-N_{\text {gals }}\right.$,
$T_{\mathrm{X}}-N_{\text {gals }}$, and $\left.T_{\mathrm{X}}-L_{\mathrm{X}}\right)$ can be minimized by excluding clusters with signs of substructure. In the left panel, we show the dependence of the scatter on the radius used for selecting cluster galaxies, while on the right we show the variation with the significance level employed. The results are shown only for the $\beta$-test. As each test has a different dependence on these parameters, their optimal values will also differ, implying that two tests could yield the same percentage of clusters with substructure through the use of very different apertures and especially significance levels. After many trials for all four tests we find that the $\beta$-test is most appropriate (for our data) for detecting substructure. It gives results that are comparable to the literature and is considered the most sensitive among the four tests (Pinkney et al. 1996). Based on Figure 8, we use a radius of $1.50 h^{-1} \mathrm{Mpc}$ and a significance level of 5\%. A similar analysis for the magnitude range suggests that our original choice $\left(m_{r}^{*}-1 \leq m_{r} \leq m_{r}^{*}+1\right)$ is appropriate.

It is interesting that only the $T_{\mathrm{X}}-N_{\text {gals }}$ relation is sensitive to the choices of radius and S.L. As noted in $\S 6$, the scatter in the $L_{\mathrm{X}}-N_{\text {gals }}$ relation is much higher than for $T_{\mathrm{X}}-N_{\text {gals }}$. The exclusion of clusters based on substructure is not sufficient to improve the $L_{\mathrm{X}}-N_{\text {gals }}$ relation. In the bottom panels of Figure 8 we show similar plots, but for the four substructure tests and only for the $T_{\mathrm{X}}-N_{\text {gals }}$ relation, using our final settings of $R=1.50 \mathrm{~h}^{-1} \mathrm{Mpc}$ and S.L. $=5 \%$. From these two panels it is clear that only the $\beta$-test is sufficiently sensitive.

The distribution of substructure results, for the four statistical tests, is shown in Figure 9. The solid lines represent the distribution of all clusters, while the thick dotted lines show the subset with substructure. A cluster is considered to have substructure if the null hypothesis is rejected at the 5\% significance level. This means that at most $5 \%$ of the Monte Carlo data sets yield a statistic more extreme than the real cluster.

We have also investigated the possibility that the substructure tests depend on the cluster contrast, richness, and redshift, with the results shown in Figure 10. As mentioned in $\S 4.1$, the contrast is defined as the ratio between the number of galaxies in the cluster region (within the same magnitude range sampled for the richness calculation) and the background error $Q_{\sigma_{\mathrm{bkg}}}$. There is no clear sign that substructure detection is contrast-dependent. The variation in the fraction of clusters showing substructure with the cluster contrast is shown in bottom panel, the redshift variation in the middle panel, and the richness dependence in the top panel. We cannot verify a clear trend with contrast, but see a mild increase with richness and a very small decrease with redshift. Since this analysis is restricted to clusters at $0.07 \lesssim z \lesssim 0.21$, it is difficult to investigate the evolution of substructure with look-back time. We postpone the study of the evolution of substructure with redshift to a future work, where we will use deeper photometry (reaching higher redshifts) combined with X-ray imaging.

The final substructure results using the 10,190 clusters at $0.07 \lesssim z \lesssim 0.21$, for each test are as follows: AST finds that $13 \%$ clusters have substructure; the $\beta$-test selects $35 \%$, the FE test finds $21 \%$, and the Lee $2 \mathrm{D}$ test finds $45 \%$, all at the $5 \%$ significance level. As we can see, AST selects by far the fewest clusters with substructure, as expected due to its lack of sensitivity. The rates found by the $\beta$ - and Lee 2D tests are in good agreement with the results of Kolokotronis et al. (2001), who found at least $\sim 45 \%$ of their clusters with strong indications of substructure. However, their sample was much smaller (only 22 clusters), and they employed other tests, using optical and X-ray data to confirm the substructure estimates. Other literature results indicate that the percentage of clusters showing substructure varies from $30 \%$ to $80 \%$. This discrepancy is generally believed to be associated with 

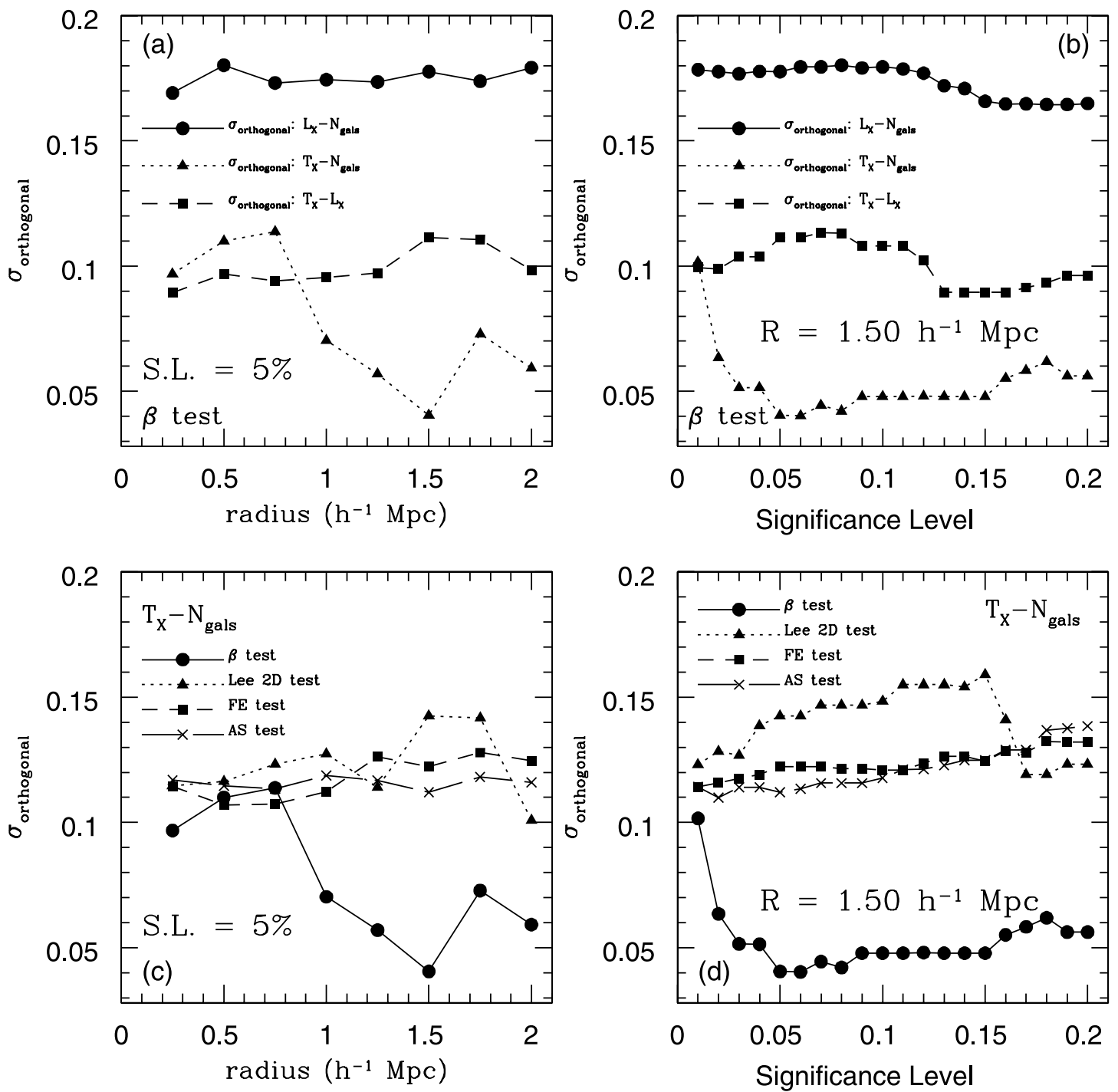

FIG. 8.-Dependence of the orthogonal scatter on the radius (left) and significance level (right) used to evaluate substructure. In the top panels we show this dependence for three relations for the $\beta$-test only. The relations used are $L_{\mathrm{X}}-N_{\mathrm{gals}}, T_{\mathrm{X}}-N_{\text {gals }}$, and $T_{\mathrm{X}}-L_{\mathrm{X}}$. In the bottom panels the dependence is shown only for the $T_{\mathrm{X}}-N_{\mathrm{gals}}$ relation for all four tests.

the method employed for each analysis (Kolokotronis et al. 2001). We stress that the variation in these results is likely also due to the cluster centroid, area, magnitude range, and significance level adopted when applying a given test. For instance, had we used $1 \%$ or $10 \%$ as our significance level for the $\beta$-test, we would find $19 \%$ and $46 \%$ of clusters with substructure, respectively. Thus, not only it is fundamentally important to compare similar data sets, the tests employed must be consistent. The same clearly holds true for comparisons with cosmological simulations.

\subsection{Correlation between Substructure and Ellipticity}

Ellipticity is generally considered to be a sign of substructure, a result that has been found by previous authors (Jones \& Forman 1999; Kolokotronis et al. 2001). We thus decided to investigate whether the substructure tests we employ in this work are correlated to ellipticity. For this purpose we used only the 445 common BAX-NoSOCS clusters at $0.07 \lesssim z \lesssim 0.21$. In order to compare our results to the ones from Kolokotronis et al. (2001), we have considered substructure measures within $0.75 h^{-1} \mathrm{Mpc}$, an aperture close to the maximum radius employed by these authors $\left(0.60 h^{-1} \mathrm{Mpc}\right)$. We have also binned the substructure and ellipticity results into substructure bins. For the four tests used in this work we fit a linear correlation of the type $\epsilon=A+B_{\text {sub }_{\text {test }}}$, where $\epsilon$ is the ellipticity value, $\operatorname{sub}_{\text {test }}$ is the result for a given substructure test, and $A$ and $B$ are the intercept and slope obtained by an orthogonal regression method (see $\S 6.2$ ). The relations obtained for all the four substructure tests are shown in Figure 11 and summarized in Table 2. We note that two of the methods (FE and Lee 2D) show a clear dependence between ellipticity and substructure. These results are in agreement to the ones found by Kolokotronis et al. (2001), although we find slopes shallower than what they found. However, it is important to keep in mind that they employed a different substructure test and selected galaxies from a smaller aperture and different magnitude range.

As can be seen from Figure 9, the $\beta$-test can take on negative values. We found that these are anticorrelated to $\epsilon$, while the positive values are correlated. We have thus considered only the positive values of $\beta$ for the results shown in Table 2 and Figure 11. From inspection of this table we see that AST is the only test that yields a slope consistent to zero. This result is consistent with the aforementioned lower sensitivity of this test (Fig. 7).

\section{OPTICAL VERSUS X-RAY CLUSTER PROPERTIES}

In this section we investigate the correlation between richness $\left(N_{\text {gals }}\right)$ and X-ray properties $\left(L_{\mathrm{X}}\right.$ and $\left.T_{\mathrm{X}}\right)$ of clusters common to DPOSS and BAX. From the 638 common clusters, 620 have $L_{X}$ 

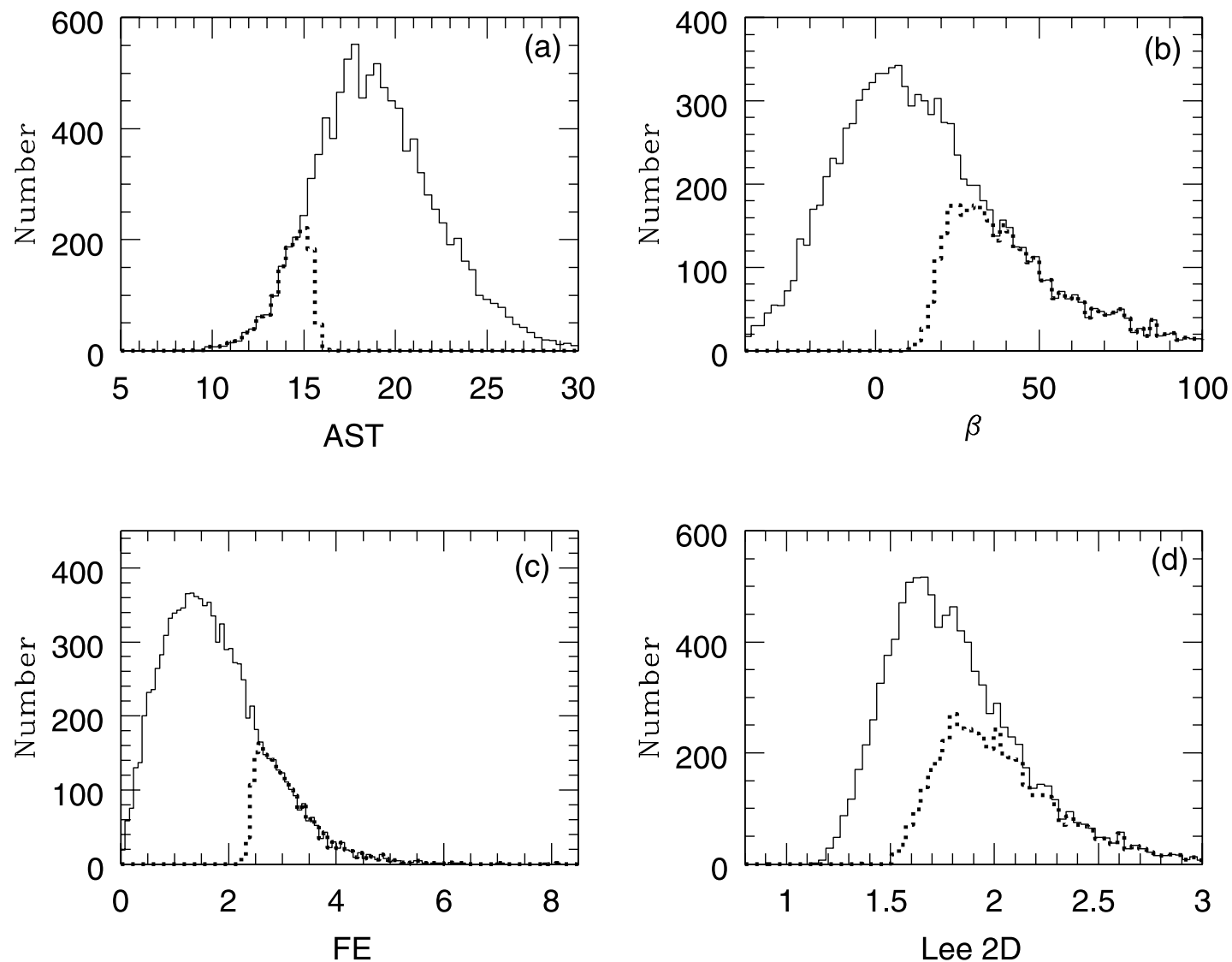

FIG. 9.-Distribution of the substructure results for the four statistical tests: $(a)$ the angular separation test (AST); $(b)$ the $\beta$-test (to improve the visualization the $\beta$-values are multiplied by 1000); (c) Fourier elongation (FE), and $(d)$ Lee 2D results. The solid lines represent the distribution of all clusters, while the thick dotted lines show the results for the subset with substructure based on the Monte Carlo simulation, rejecting the null hypothesis at the $5 \%$ significance level. Note that AST is the only test for which lower values represent a more extreme statistic. For the FE test we show the distribution of all clusters considered to have substructure at the $5 \%$ significance level, without further excluding clusters with FE $>2.5$. As we can see the adoption of this additional criterion — as suggested by Pinkney et al. (1996) — excludes only a small additional number of clusters.

(of which two have no richness estimate), while $T_{\mathrm{X}}$ is available for 101 clusters. As mentioned before, for analyses involving substructure estimates we restrict the study to $0.07 \lesssim z \lesssim 0.21$, reducing the original samples from 618 to 430 clusters (with $L_{\mathrm{X}}$ and richness determined) and from 101 to 53 systems (with $T_{\mathrm{X}}$ ). Clusters with $N_{\text {gals }}<10$ are also excluded when establishing these correlations (see the discussion in $\S 4.1$ and Fig. 3) and a $3 \sigma$ clipping is also applied to exclude outliers. We also discuss how the scatter in the established relations is affected by the radius adopted for calculating richness, the redshift difference $(\Delta z)$ between BAX and DPOSS, positional offsets, the contrast of the cluster, and the presence of substructure.

\subsection{Dependence on Input Data}

We reiterate that the orthogonal scatter $(\sigma)$ and the linear correlation coefficient $\rho$ of the $L_{\mathrm{X}}-N_{\text {gals }}$ and $T_{\mathrm{X}}-N_{\text {gals }}$ relations are extremely sensitive to the aperture used for calculating richness (see Fig. 2). These results are in good agreement with those of Popesso et al. (2004), who verified the dependence of the scatter in the $L_{\mathrm{X}}-L_{\mathrm{opt}}$ relation on the aperture used. When using very small apertures to estimate $N_{\text {gals }}$ the galaxy number count becomes very uncertain due to centroid errors. For large radii the background contribution grows rapidly, contaminating the richness estimates. As explained in $\S 4.1$ we use a $0.50 h^{-1} \mathrm{Mpc}$ radius for estimating $N_{\text {gals }}$ for comparisons to X-ray properties.

The effects of $\Delta z$ and positional offset on the scatter in the $L_{X^{-}}$ $N_{\text {gals }}$ relation are shown in the left and right panels of Figure 12, respectively. As before, we adopt the BAX redshift to calculate $N_{\text {gals }}$, since the richness is strongly redshift-dependent (through the apparent radius and the magnitude range). This should minimize the scatter in the $L_{\mathrm{X}}-N_{\text {gals }}$ relation, and we expect this scatter to show no dependence on $\Delta z$ if the redshift errors are random. The variation shown in the left panel of Figure 12 is small $(\sim 0.020)$ but real, and is probably due to the fact that the BAX redshifts are not homogeneous measures. They are automatically retrieved from NED, and are most often spectroscopic, but some are photometric estimates. Even the spectroscopic redshifts may be based on only one or two galaxy spectra. Thus, we conclude that even after adopting the BAX redshift, we could further reduce the scatter in the $L_{\mathrm{X}}-N_{\text {gals }}$ relation by restricting the sample to clusters with small values of $\Delta z$ (at the cost of reducing the sample size). The improvement in the least-squares fit of the $L_{\mathrm{X}}-N_{\text {gals }}$ relation for clusters with small positional offset is expected. The dependence of $\sigma_{\text {orthogonal }}$ is of the same order as that shown in the left panel.

We also investigate the dependence of the $L_{\mathrm{X}}-N_{\text {gals }}$ with the minimum and maximum redshifts used to select clusters for the fit. The results are optimal for clusters at $0.15 \leq z \leq 0.30$, with strong sensitivity to the lower redshift limit. This is because most of the low-richness systems are at low redshift, and these poor clusters have the largest richness errors (Fig. 3). Based on Figures 2 and 12 we use a radius of $0.50 h^{-1}$ Mpc for calculating richness, but do not apply any cut with redshift or offset. All the dependences of $\sigma_{\text {orthogonal }}$ on the redshift cutoff, $\Delta z$ and the 


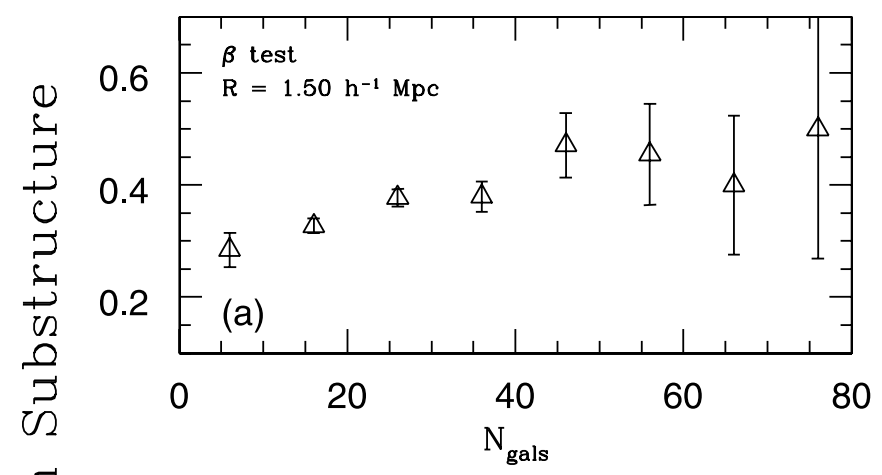

the comment of Isobe et al. (1990) concerning linear regression methods that the two methods used here (and others available in the literature) find regression coefficients that are theoretically different from each other. In other words, they do not represent different estimates of the same quantity. Even if the entire population were sampled, the measured slopes would differ (only in special cases is a single relation obtained, such as when $\rho=1)$.

In order to evaluate which of these methods is best suited for our purposes, we generated artificial data sets with known scatter and slopes. The relations resulting from the optical-X-ray comparison could be very steep and noisy, especially those involving $L_{\mathrm{X}}$. Hence, we created data sets of 1000 points for different angles (ranging from $1^{\circ}$ to $89^{\circ}$ ) and for 20 different scatters (from 0.01 to 0.40 ). We then run slopes and bces-regress for the bisector and orthogonal solutions, computing the difference between the input and output slopes $\left(\left|\Delta_{\text {slope }}\right|\right)$. This procedure is repeated 100 times. The results are shown in Figure 13, where the error bars for each point represent the standard deviation for the 100 events. In the top panels we show the variation of $\left|\Delta_{\text {slope }}\right|$ with angle (at a fixed scatter of 0.20 ), while the dependence with scatter is shown in the bottom panels (for a fixed angle of $65^{\circ}$ ). The tests using unweighted and weighted fits are shown in the left and right panels, respectively. The bisector results are plotted as solid lines, while the orthogonal solutions are plotted as dotted lines. As with the real data, a $3 \sigma$ clipping is applied to exclude outliers. The orthogonal minimization is clearly superior for recovering the input slopes, especially when there is large scatter. If we do not eliminate outliers, the results shown in the top panels diverge for large angles, but the bisector solution still shows larger deviations from the original slopes. Given these results, we use the orthogonal regression for all fits. We note that some results in the literature (Markevitch 1998) have used the bisector, which may be misleading.

\subsection{The X-Ray and Optical Relations \\ 6.3.1. X-Ray Luminosity and Temperature}

Fig. 10.- Substructure results for the $\beta$-test as a function of cluster contrast (bottom panel), redshift (middle panel), and richness (top panel).

positional offset are much less pronounced than the dependence on aperture. Thus, we do not restrict the sample based on these weaker effects, which would significantly reduce the number of clusters and make it difficult to quantify the most significant effects. The impact of contrast and substructure are investigated further below.

\subsection{Dependence on Fitting Method}

Before examining the connection between optical and X-ray parameters we investigate which linear regression solution is more robust, using the packages slopes and bces-regress from Isobe et al. (1990) and Akritas \& Bershady (1996), respectively. The first performs unweighted fits, while the second considers errors as well as intrinsic scatter. Because BAX provides no error estimate for $L_{\mathrm{X}}$, we perform an unweighted fit (using slopes) for the $L_{\mathrm{X}}-N_{\text {gals }}$ relation, while bces-regress is used for $T_{\mathrm{X}}-N_{\text {gals }}$. We use two commonly used solutions for each package. The first is the ordinary least-squares (OLS) bisector solution, the line which bisects the $\operatorname{OLS}(Y \mid X)$ (minimizing the residuals in $Y$ ) and $\operatorname{OLS}(X \mid Y)$ (minimizing the residuals in $X$ ) lines. The second solution is the orthogonal regression line, which minimizes the perpendicular distances (Isobe et al. 1990; Feigelson \& Babu 1992; Akritas \& Bershady 1996). We stress

We begin our analysis by examining the $L_{X}-T_{X}$ relation (Fig. 14) derived from the BAX data, to verify the reliability of this data source for our purposes. We use 120 clusters with available temperatures within the NoSOCS region. We show the best fit using all 120 clusters (two are excluded as outliers) as the solid thin line. We show the results obtained using only the 100 clusters with optical contrast $\geq 10.0$ as a thin dotted line. As discussed below, the scatters and slopes of the scaling relations depend on the contrast cut applied to the cluster sample. Interestingly, the optical contrast appears to provide a useful test for selecting clusters to affect the $L_{\mathrm{X}}-T_{\mathrm{X}}$ relation. Literature results are shown by thick lines. The solid and dotted lines show the results from Markevitch (1998), with the dotted line for $L_{\mathrm{X}, \text { bol }}$. We note that Markevitch (1998) employed a bisector method for fitting this relation. If we also use the bisector slope, our results are considerably less steep $\left(L_{\mathrm{X}} \propto T_{\mathrm{X}}^{2.71}\right)$, showing better agreement with Markevitch (1998). However, as discussed above, the bisector is not the optimal method for fitting steep slopes especially in the presence of large scatter. The results from David et al. (1993) are plotted as the dotted thick line.

\subsubsection{X-Ray Luminosity and Richness: Structure Segregation}

The $L_{\mathrm{X}}-N_{\text {gals }}$ relation is shown in Figure 15, along with the effects of the contrast and the presence of substructure in the derived relation. The comparison of all clusters at $0.07 \lesssim z \lesssim 0.21$ is exhibited in the bottom left panel, while the high-contrast 

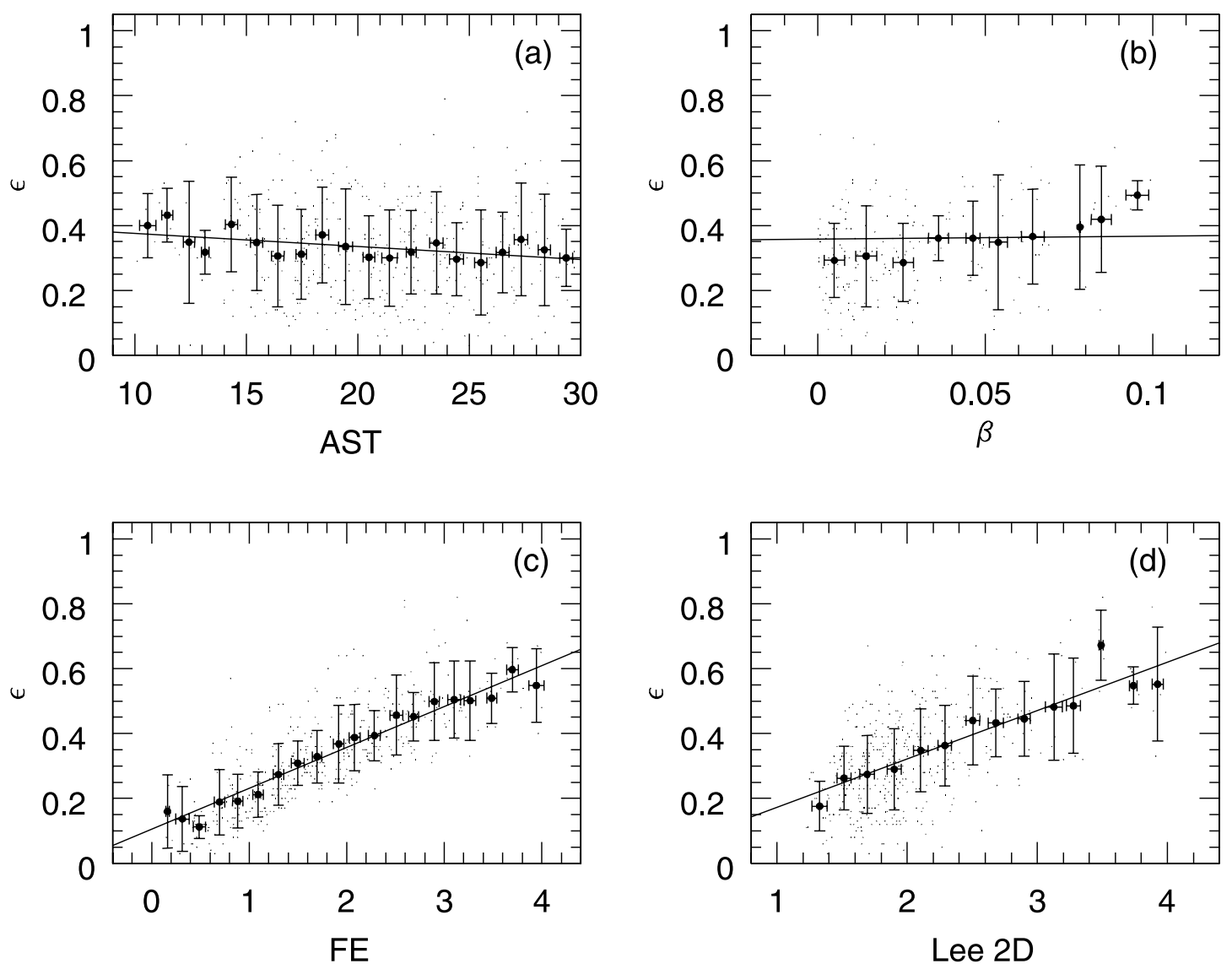

FIG. 11.-Correlation between ellipticity and substructure for the four statistical tests: (a) the angular separation test (AST); (b) the $\beta$-test; $(c)$ Fourier elongation (FE), and $(d)$ Lee $2 \mathrm{D}$ results.

systems (contrast $\geq 12.0$ ) are shown in the bottom right. The substructure-free clusters are exhibited in the top left panel and those with substructure in the top right. The fit shown by a solid line in all panels is that obtained for the substructure-free clusters. In Figures $15 b, 15 c$, and $15 d$ we also show the $3 \sigma$ boundaries of this fit (dotted lines) and the fit obtained with the data shown in each panel (all clusters, high-contrast systems, and clusters with substructure) with the dashed line. In all panels we indicate the number of clusters used in the fits (after excluding outliers at a $3 \sigma$ level), the linear correlation coefficient $(\rho)$, the intercept $(A)$, the slope $(B)$, and the orthogonal scatter of the relation obtained for that sample.

From this figure we see that the scatter changes only slightly when selecting only substructure-free clusters, while it is reduced by $\sim 12 \%$ when selecting high-contrast systems. The combination of the two criteria (substructure and contrast) does not improve the scatter compared to applying only the contrast cut. Interestingly, the slope is significantly higher for the high-contrast clusters. Popesso et al. (2004) also found improvement in the relations

TABLE 2

Correlation between Ellipticity and Substructure

\begin{tabular}{|c|c|c|c|}
\hline Substructure Test & $\begin{array}{l}\text { Linear Correlation } \\
\text { Coefficient }\end{array}$ & Intercept & Slope \\
\hline$\beta$ & 0.92 & $0.358 \pm 0.017$ & $0.092 \pm 0.052$ \\
\hline Lee 2D ................... & 0.94 & $0.023 \pm 0.037$ & $0.149 \pm 0.017$ \\
\hline FE & 0.98 & $0.105 \pm 0.014$ & $0.126 \pm 0.007$ \\
\hline 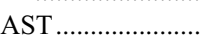 & -0.59 & $0.415 \pm 0.025$ & $-0.004 \pm 0.001$ \\
\hline
\end{tabular}

when excluding low-contrast systems. We conclude that the scatter in the $L_{\mathrm{X}}-N_{\text {gals }}$ relation is mainly due to the difficulty of obtaining accurate measures of these parameters for low-luminosity systems. If there is any difference for clusters with or without substructure, it is washed out in these relations. Thus, when correlating $L_{X}$ to the optical parameters we do not detect any significant structure segregation.

\subsubsection{X-Ray Temperature and Richness: Structure Segregation}

The $T_{\mathrm{X}}-N_{\text {gals }}$ relation is plotted in Figure 16 (with $3 \sigma$ outliers shown as open symbols), in a manner analogous to the previous figure. In the bottom left panel of Figure 16 we show the results for the 53 clusters at $0.07 \lesssim z \lesssim 0.21$ with $T_{\mathrm{X}}$ and richness measured. As before, in the bottom right panel we show the results for the high-contrast systems (contrast $\geq 12.0$ ). In the top left the sample includes only clusters found to have no substructure by the $\beta$-test at the $5 \%$ S.L, while in the top right we show the complementary clusters. A few things stand out from this figure. First, $T_{\mathrm{X}}$ is clearly better correlated with the optical parameters than $L_{\mathrm{X}}$. Second, substructure has an effect on the scatter of the $T_{\mathrm{X}}-N_{\text {gals }}$ relation, which was not seen when using $L_{\mathrm{X}}$. When excluding clusters with substructure the scatter of the $T_{\mathrm{X}}-N_{\text {gals }}$ relation is reduced by $\sim 63 \%$. Third, there is no improvement when applying the contrast cut. This is mainly due to the fact that $T_{\mathrm{X}}$ is typically available only for the most massive systems, so the analysis is restricted to a small range of temperature (and also contrast). Of the 430 clusters with $L_{X}$ measured, $177(\sim 41 \%)$ clusters are low-contrast systems, while they are only $14(\sim 26 \%)$ of the 53 with $T_{\mathrm{X}}$. Finally, when combining the two criteria (substructure and contrast) the scatter is not very different from if we employ 


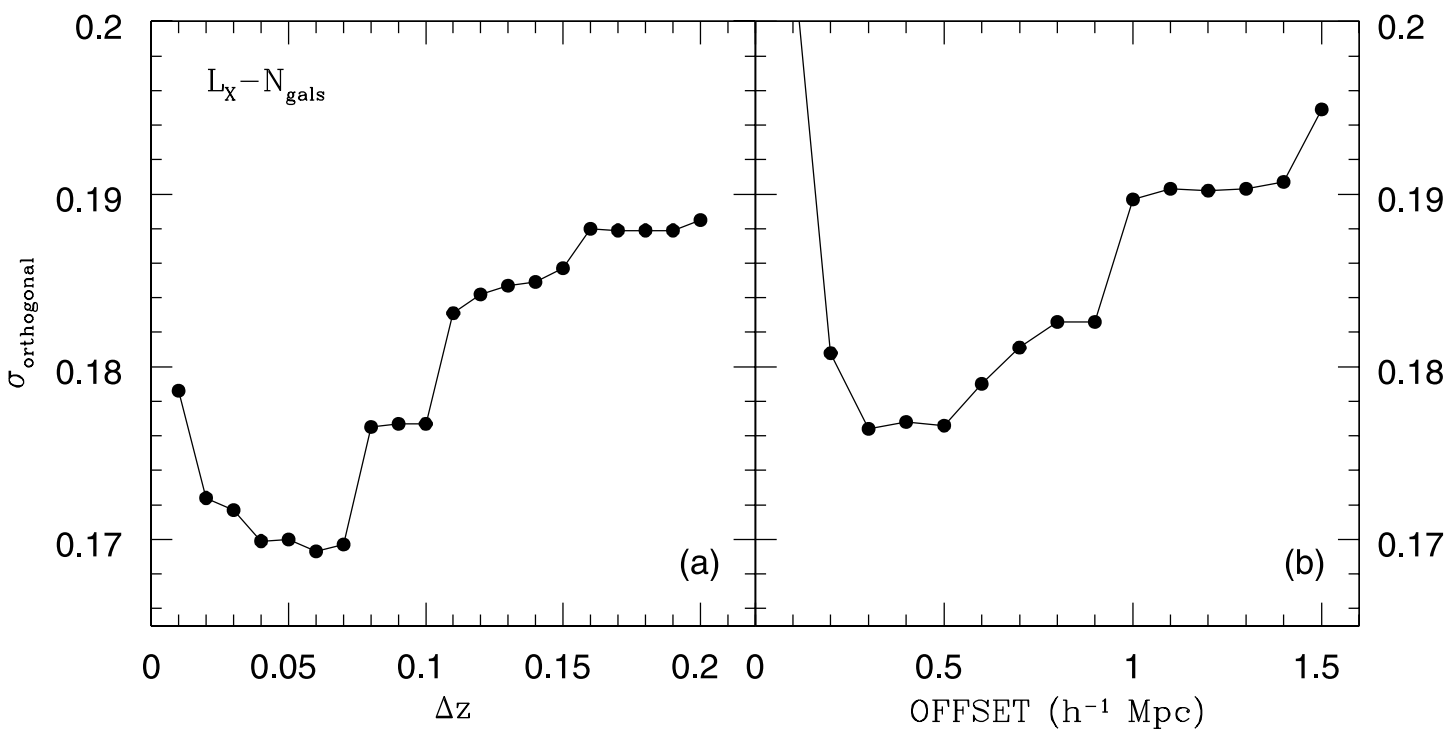

FIG. 12.- Variation of the scatter of the $L_{\mathrm{X}}-N_{\text {gals }}$ relation as a function of the maximum absolute redshift difference between the NoSOCS and BAX values (left panel) and the positional offset (right panel).

only the substructure cut, implying that the dominant factor in the scatter of this relation is substructure.

The small number of clusters with available temperatures is certainly a problem when establishing these relations and applying the above cuts. However, Smith et al. (2005) have also recently found that the scatter in the scaling relations between cluster mass, X-ray luminosity, and temperature is dominated by unrelaxed clusters. Their sample is smaller than ours (and they are also limited to massive systems), but serves to corroborate the current results. An opposite result (no structure segregation
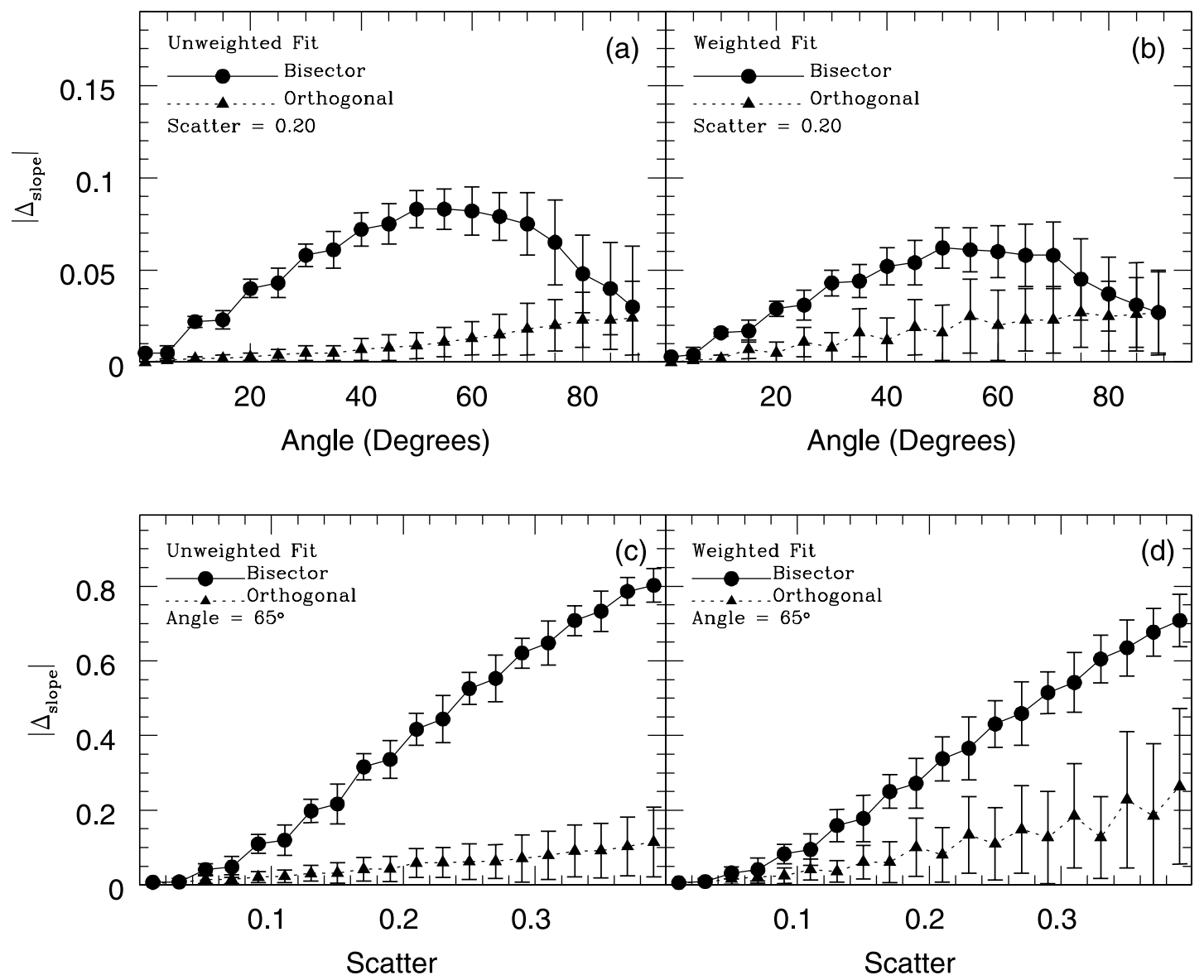

FIG. 13.-Variation of $\mid \Delta_{\text {slope }}$, the difference between the input slope and the one recovered by the bisector and orthogonal solutions. In the top panels we show the variation

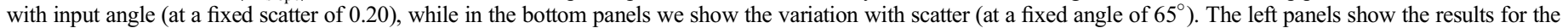
unweighted fits and the right panels for the weighted fits. The bisector results are given by the solid lines, while the orthogonal ones are shown by the dotted lines. 


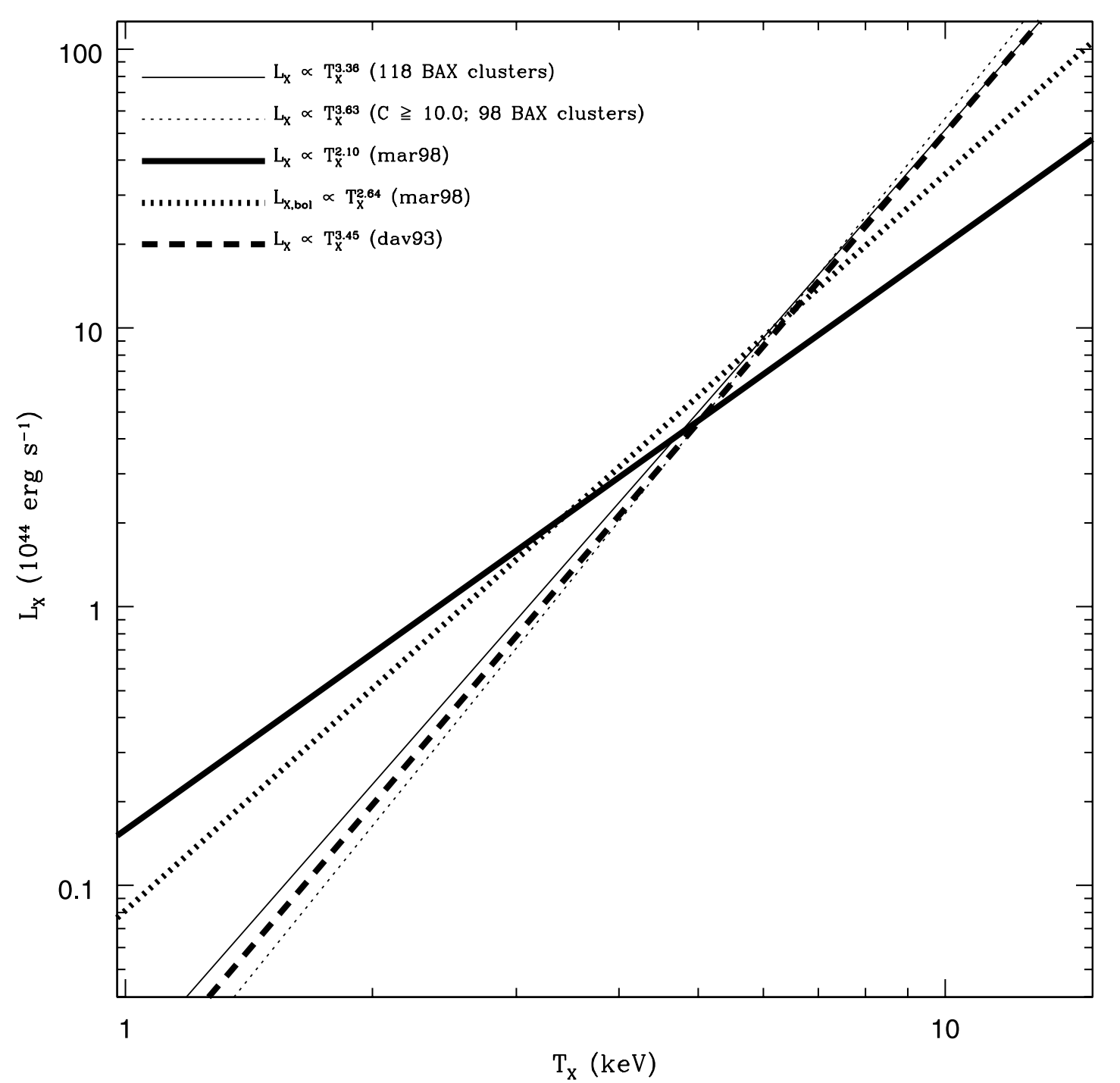

FIG. 14. $-L_{\mathrm{X}}-T_{\mathrm{X}}$ relation obtained using all $120 \mathrm{BAX}$ clusters (with two excluded as outliers) in the NoSOCS region is shown as a thin solid line. The sample is then restricted to high optical contrast systems $(C \geq 10.0)$ with the result indicated by the thin dotted line. Literature results are indicated by thick lines. The relations obtained by Markevitch (1998) are indicated by the solid and dotted lines (the last one uses bolometric luminosities). The dashed line indicates the results from David et al. (1993). [See the electronic edition of the Journal for a color version of this figure.]

in the scaling relations) is found by O'Hara et al. (2006), using both observational data and simulations. They compare the deviations of clusters from the best-fit relations (obtained for all systems and not for the separate sets with or without substructure) for different scaling relations and find no evidence for higher scatter for clusters with more substructure. The substructure tests they employ are different than the ones used here. Their method for dividing the sample into clusters with or without substructure is also unclear (there is no cut at a given significance level). However, the most important differences between our methodology and theirs are the fact that they investigate substructure with an aperture that scales with the cluster mass $\left(R_{500}\right.$ and $\left.R_{2500}\right)$, while we use a fixed metric $\left(1.50 h^{-1} \mathrm{Mpc}\right.$ radius $)$, and that they rely on $\mathrm{X}$-ray data, while we use optical data. Clusters with more substructure are probably dynamically younger and would thus be expected to deviate from relations established for well-relaxed systems. In future work, we will investigate substructure using these alternative approaches (O'Hara et al. 2006) to see whether our current results persist.

It comes as no surprise that the X-ray temperature correlates better with optical properties than the X-ray luminosity. $T_{\mathrm{X}}$ has been shown to provide a much tighter correlation to cluster mass than X-ray luminosity (Voit 2005), as $T_{\mathrm{X}}$ is closely related to the cluster's potential well. What we find here is that the connection between richness (which is a mass tracer) and $T_{\mathrm{X}}$ has a smaller scatter than if we used $L_{X}$. Similar results are found by Yee $\&$ Ellingson (2003) and Popesso et al. (2005), who find tighter relations between their richness estimates $\left(B_{\mathrm{gc}}\right.$ or $\left.L_{\mathrm{opt}}\right)$ and $T_{\mathrm{X}}$ than with $L_{\mathrm{X}}$. We also note that samples constructed purely at one wavelength may not include the same types of objects (Lubin et al. 2004).

\subsubsection{X-Ray Luminosity and Richness: Sample Dependence}

We now compare our findings to literature results. We show in Figure 17 the connection between $L_{\mathrm{X}}$ and richness or $L_{\mathrm{opt}}$. Our results are shown by thin lines, while the literature ones are thick lines. When performing this comparison we investigate a few effects: (1) how the slope of the optical versus X-ray relation changes with cluster contrast; (2) the impact of the centroid (optical or $\mathrm{X}$-ray) on the slope; (3) the slope difference when using richness or $L_{\text {opt }}$ as the optical parameter; (4) the difference in the results obtained by the bisector and orthogonal fits. All of the relations 


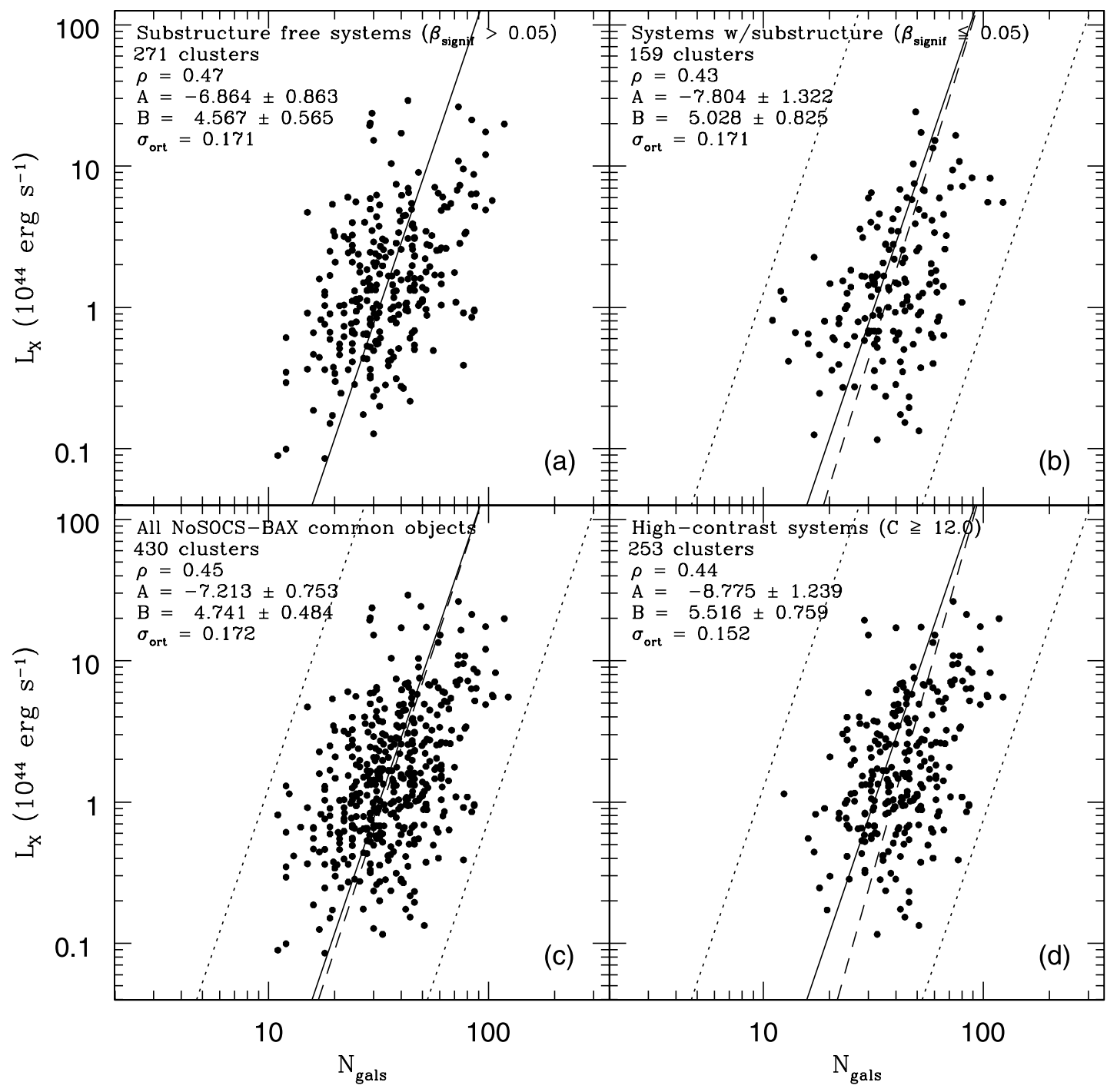

FIG. 15. $-L_{\mathrm{X}}-N_{\text {gals }}$ relation is shown for $(a)$ all clusters at $0.07 \lesssim z \lesssim 0.21,(b)$ clusters with contrast $\geq 12.0,(c)$ clusters without substructure (at the significance level of $5 \%$ ) based on the $\beta$-test, and $(d)$ clusters with substructure according to the $\beta$-test. The fit shown in all panels (solid lines) is the orthogonal solution obtained for the sample in the top left panel. For three panels $(a, b$, and $d)$ the dotted lines show the $3 \sigma$ boundaries for this fit. For these three panels $(a, b$, and $d)$ the fit obtained with the data shown on each panel (all clusters, high contrast systems and clusters with substructure) is shown by the dashed line.

based on our data shown here include all clusters (we do not exclude clusters with $N_{\text {gals }}<10.0$, the only exception being the relation for clusters with no substructure) and are obtained using an orthogonal regression. We also stress that the relations based on the X-ray center (BAX) are derived from all BAX clusters within the area covered by NoSOCS (whether or not they have a counterpart in DPOSS). The relations obtained with the optical center include only clusters common to the optical and X-ray catalogs. Hence, the former relations are based on larger samples of clusters.

Figure 17 shows the $L_{\mathrm{X}}-N_{\text {gals }}$ relations for a variety of samples. All of the results from our sample are plotted as thin lines, with the following meanings:

1. Solid line.-All BAX clusters in the NoSOCS region. Of the 792 BAX clusters, 744 have both richness and $L_{X}$ available. Ten clusters are eliminated as outliers after a $3 \sigma$ clipping is applied.

2. Dotted line.-The previous sample trimmed to clusters with optical contrast $\geq 5$.0.

3. Short-dashed line.-The sample is further reduced to clusters with contrast $\geq 10.0$. For the above three relations we use the X-ray centroid when estimating $N_{\text {gals }}$.
4. Long-dashed line.- Same as the solid line above, but using only the common clusters between NoSOCS and BAX (with available $L_{\mathrm{X}}$ and $N_{\mathrm{gals}}$ ), and the optical centroid for the richness calculation.

5. Dash-dotted line.-The sample is then cut to consider only substructure free systems, using the optical centroid (as shown in Fig. 15).

6. Long-dash-dotted line.-The $L_{\mathrm{X}}-L_{\mathrm{opt}}$ relation, using the $\mathrm{X}$-ray centroids.

We also show a variety of results from the literature as thick lines:

1. Solid line.-The $L_{\mathrm{X}}-L_{\mathrm{opt}, i}$ relation obtained by Popesso et al. (2004) for their full sample. We note that they too use the X-ray centers.

2. Dotted line.-The relation from Popesso et al. (2004) when groups are excluded.

3. Short-dashed line.-The correlation between $L_{\mathrm{X}}$ (bolometric) and optical richness (given by $\Lambda_{\mathrm{cl}}$ ) from Donahue et al. (2001).

4. Long-dashed line.-The correlation between $L_{\mathrm{X}}$ and richness $\left(B_{\mathrm{gc}}\right)$ obtained by Yee \& Ellingson (2003). 


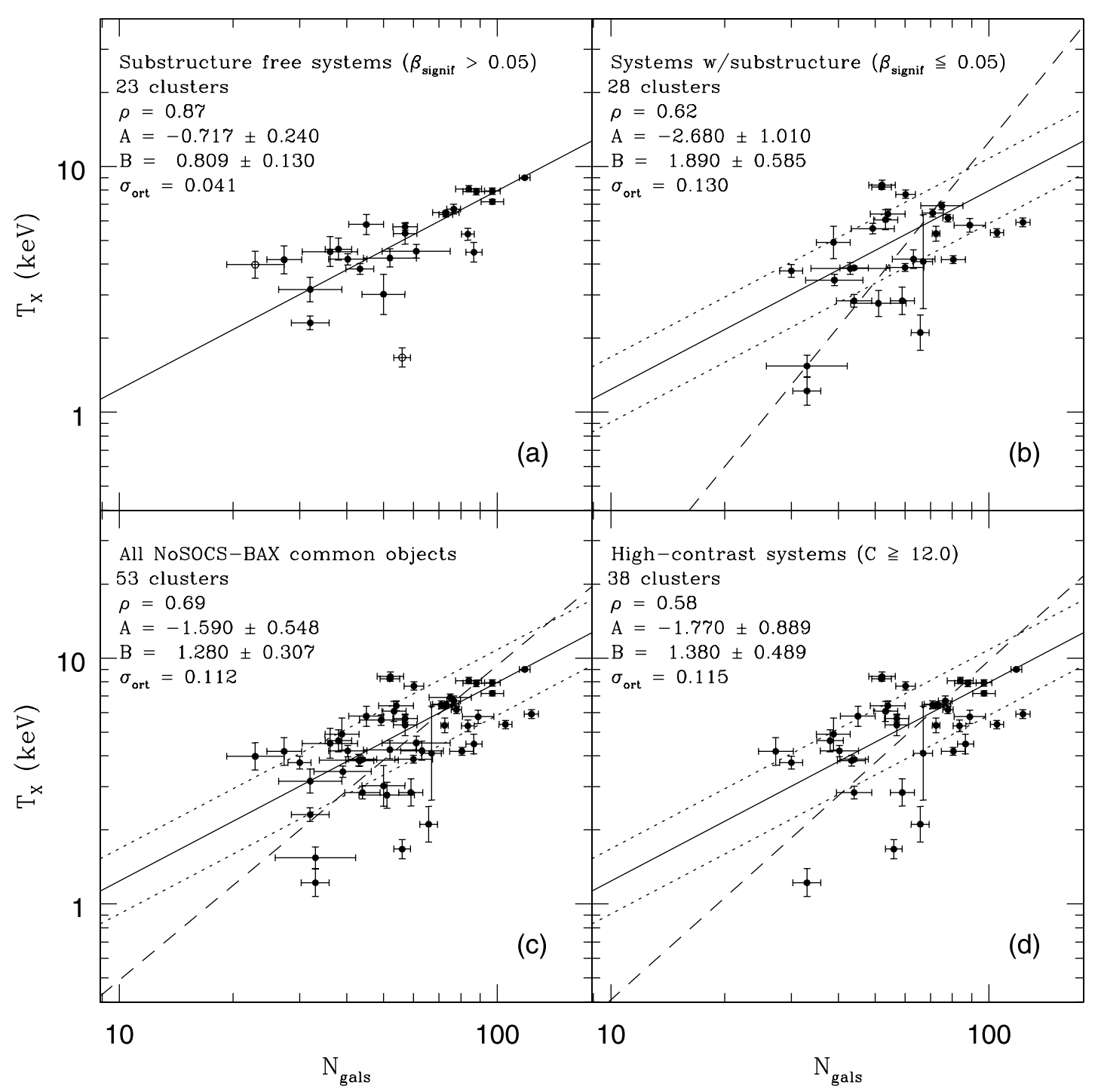

FIG. 16. $-T_{\mathrm{X}}-N_{\text {gals }}$ relation for all clusters at $0.07 \lesssim z \lesssim 0.21$. The full sample is shown in the bottom left panel; high-contrast systems are exhibited in the bottom right panel; clusters considered to be substructure free are exhibited in the top left panel, where two outliers are shown with an open symbol; clusters with substructure are shown in the top right panel.

We note that Yee \& Ellingson (2003) use a bisector solution, which is generally less steep. If we considered a bisector solution in our analysis the powers in the relations we obtained (thin lines) would range from 1.79 to 2.19 , which is in good agreement with their results.

From this figure we can see that the slope increases rapidly as we consider higher contrast systems. This trend was already evident in Figure 15, where we also see that the scatter is greatly reduced when the sample is restricted. However, this behavior does not persist for much larger values of the contrast cut. In Figure 17 we show the results for the full sample and with cuts at $C \geq 5.0$ and 10.0 . If we increase the cut to $C \geq 15.0$ or 20.0 the slope variation ceases, with the slope actually becoming lower. That is due to sampling effects as the number of clusters is drastically reduced. It is important to mention that contrast is directly proportional to richness. Thus, if richness cuts were appliedinstead of contrast cuts - we would find similar trends. An important issue is that when using low-luminosity or low-richness systems the data is likely to be censored. Donahue et al. (2001) extensively discuss this problem and show how the correlation between $L_{\mathrm{X}}$ and richness $\left(\Lambda_{\mathrm{cl}}\right)$ changes when the fit is derived in conjunction with the $\Lambda_{\mathrm{cl}}$ function and the X-ray luminosity function (i.e., taking into account sampling effects at low-flux regimes).

Based on Figure 17, a very interesting conclusion is that the relations are less steep when using the X-ray centroid (instead of optical) to calculate richness (or $L_{\text {opt}}$ ). When using all clusters and considering the $\mathrm{X}$-ray center, the slope is 3.73 , increasing to 4.29 and 5.60 when trimming the sample at $C \geq 5.0$ or 10.0 , respectively. The slopes measured when considering the optical centroid for these three cases (using the common BAX and NoSOCS clusters) are 4.54, 5.09, and 6.40. This effect is associated to the contrast cuts, as the galaxy number density is generally reduced when we consider the X-ray centroid, in which case we expect the slopes to be smaller. We also find that the relation using optical luminosity is shallower than the one with richness. When comparing our results to the literature we note that Popesso et al. (2004) found the same effect regarding the contrast cut (by excluding groups from their main sample). The relations we obtain are also in good agreement to their results, as well as those from Donahue et al. (2001) and Yee \& Ellingson (2003). In the latter case there is concordance if we consider only the bisector solution. 


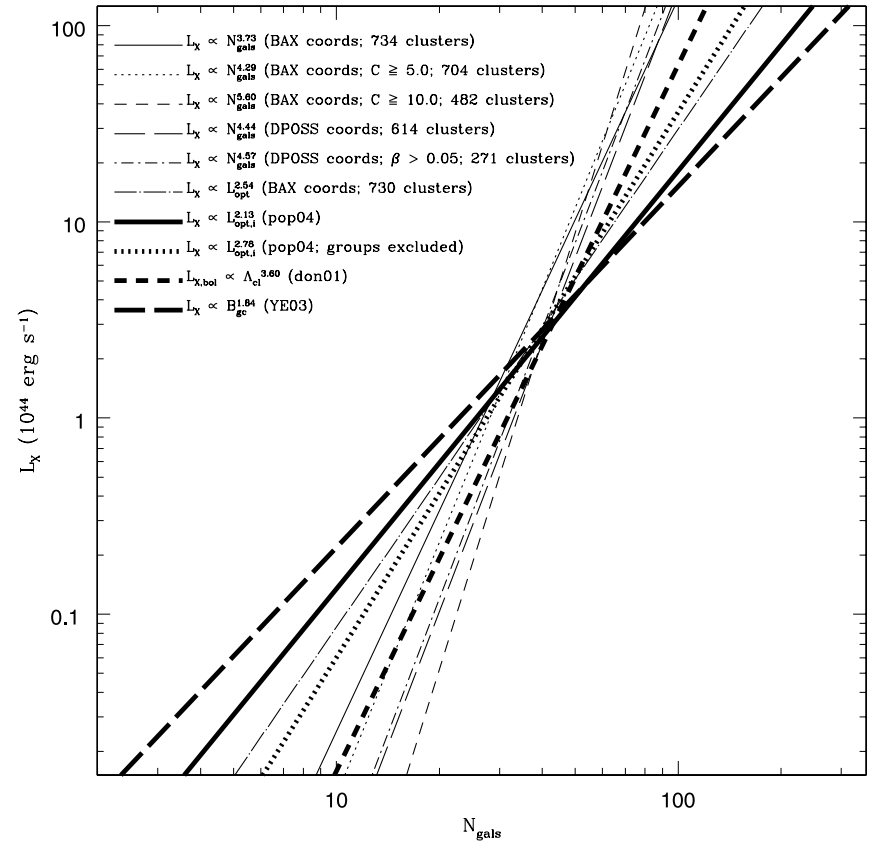

Fig. 17.-Correlation between $L_{\mathrm{X}}$ and richness or $L_{\text {opt }}$. The thin lines show the results obtained in this work, while the thick lines exhibit results from the literature. This work: (1) $L_{\mathrm{X}}-N_{\text {gals }}$ for all BAX clusters in the NoSOCS region (solid line); (2) the previous sample trimmed at contrast $\geq 5.0$ (dotted line); and (3) the sample is further cut at contrast $\geq 10.0$ (short-dashed line). For relations $1-3$ we considered the X-ray centroid when estimating $N_{\text {gals }}$; (4) same as (1), but considering all common clusters between NoSOCS and BAX and using the optical centroid for richness calculation (long-dashed line); (5) sample 4 cut to consider only substructure free systems (short-dash-dotted line); and (6) the connection between $L_{\mathrm{X}}$ and $L_{\mathrm{opt}}$ (BAX coordinates are used for computing $L_{\mathrm{opt}}$ ) for all BAX systems in the NoSOCS region (long-dash-dotted line). Literature results: (7) the $L_{\mathrm{X}}-L_{\mathrm{opt}, i}$ relation obtained by (Popesso et al. 2004) for their full sample (solid line); (8) the relation obtained by the same authors when excluding groups (dotted line); (3) $L_{\mathrm{X}}$ (bolometric) vs. optical richness $\left(\Lambda_{\mathrm{cl}}\right)$ from Donahue et al. (2001; shortdashed line); and (4) the connection between $L_{\mathrm{X}}$ and richness $\left(B_{\mathrm{gc}}\right)$ as obtained by Yee \& Ellingson (2003; long-dashed line). [See the electronic edition of the Journal for a color version of this figure.]

We define $N_{\text {gals }}$ differently from the richness parameters in the literature shown here $\left(\Lambda_{\mathrm{cl}}\right.$ and $\left.B_{\mathrm{gc}}\right)$. Thus, the most direct comparison of our results to the literature is made when considering the relations involving $L_{\mathrm{opt}}$. The results from Popesso et al. (2004) show less power than ours (2.13 in comparison to 2.54). However, their sample may be composed of a larger number of low-luminosity systems (groups) than ours. When they exclude groups, the power increase to 2.78 , in much better agreement with our findings. Even considering their full sample our results agree at the $2 \sigma$ level, while the agreement is at the $1 \sigma$ level for their high-contrast sample. The use of the optical center (instead of the $\mathrm{X}$-ray centroid) for computing $L_{\text {opt }}$ yields steeper relations. When using all the common BAX-NoSOCS clusters we find a slope of 3.01 for the $L_{\mathrm{X}}-L_{\mathrm{opt}}$ relation, which disagrees with the full sample of Popesso et al. (2004) at the $3 \sigma$ level, but is in concordance - at the $2 \sigma$ level- to their results excluding groups.

Another effect that merits attention is the fitting method employed (see $\S 6.3$ ). Literature results are often contradictory, and one of the causes that is rarely discussed is the linear regression employed. We have shown (Fig. 14) that our results differ from Markevitch (1998), mostly because they adopt a bisector solution when fitting the $L_{\mathrm{X}}-T_{\mathrm{X}}$ relation. On the other hand our, findings are consistent with those of David et al. (1993). Similar differences are shown when comparing X-ray luminosity and richness. The smaller power obtained by Yee \& Ellingson (2003) in comparison to ours is mainly associated with the fitting method (bisector

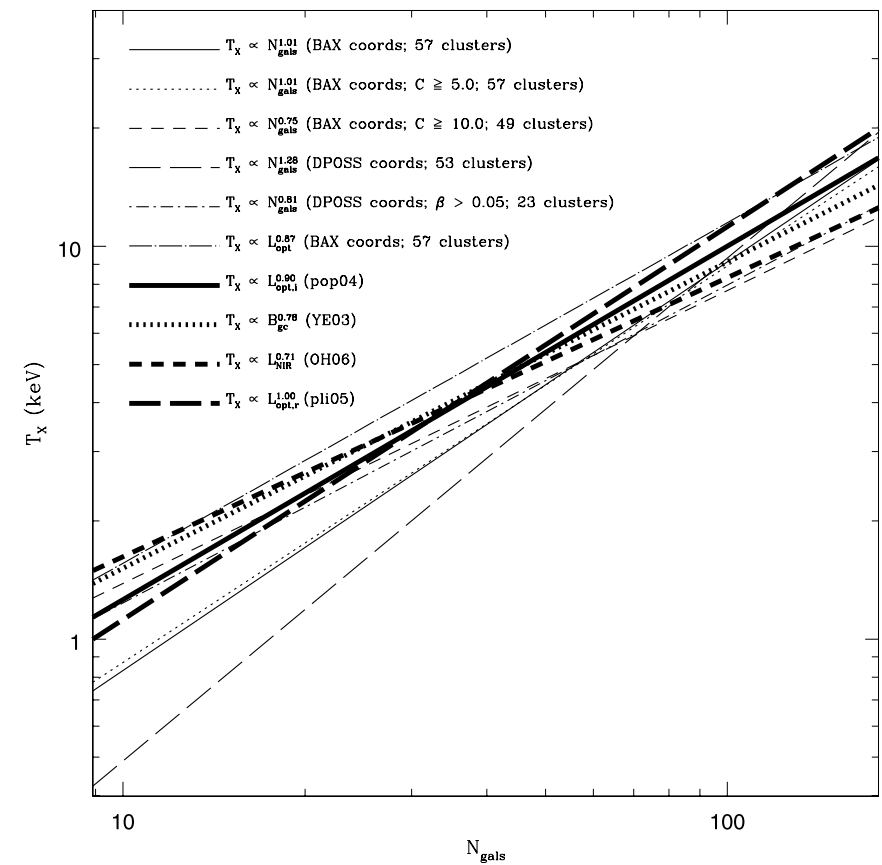

Fig. 18.-Connection between temperature and richness or optical luminosity. The thin lines show the results obtained in this work, while the thick lines exhibit results from the literature. The meaning of the thin lines is analogous to the previous figure, but now for the $T_{\mathrm{X}}-N_{\text {gals }}$ relation and only considering clusters at $0.07 \lesssim z \lesssim 0.21$. The literature results (thick lines) are given for the connection between $T_{\mathrm{X}}$ and $L_{\mathrm{opt}, i}$ obtained by Popesso et al. (2004, solid line); the $T_{\mathrm{X}}-B_{\mathrm{gc}}$ relation obtained by Yee \& Ellingson (2003; dotted line); the $T_{\mathrm{X}}-L_{\mathrm{NIR}}$ correlation from O'Hara et al. (2006; short-dashed line); and the $T_{\mathrm{X}}-L_{\mathrm{opt}, r}$ correlation from Plionis et al. (2005; long-dashed line). [See the electronic edition of the Journal for a color version of this figure.]

in their case). It is necessary to consider all of the possible effects (centroid adopted, flux limit, and fitting method, at a minimum) when attempting to calibrate a mass-observable relation. Systematic errors in the measurement of cluster mass are exponentially amplified by the steepness of the cluster mass function. In future work we will link richness to mass and then quantify the effects of different solutions on the mass function estimates.

\subsubsection{X-Ray Temperature and Richness: Sample Dependence}

In Figure 18 we show the connection between $T_{\mathrm{X}}$ and richness or $L_{\text {opt }}$. As before, our findings are shown by thin lines, while thick lines show the literature results. We have also not excluded clusters with $N_{\text {gals }}<10.0$ (except for the relation of clusters without substructure), and the fits are obtained through orthogonal regression. The results shown here include only clusters at $0.07 \lesssim z \lesssim 0.21$. The thin lines (our work) are analogous to those in the previous figure, but for the relations involving $T_{\mathrm{X}}$. The literature results (thick lines) are the $T_{\mathrm{X}}-L_{\mathrm{opt}, i}$ relation obtained by Popesso et al. (2004; solid line), the $T_{\mathrm{X}}-\mathrm{B}_{\mathrm{gc}}$ relation from Yee \& Ellingson (2003; dotted line), the $T_{\mathrm{X}}-L_{\mathrm{NIR}}$ relation from O'Hara et al. (2006; short-dashed line), and the $T_{\mathrm{X}}-L_{\mathrm{opt}, r}$ relation obtained by Plionis et al. (2005) (long-dashed line). We do not find the same trend with contrast as for $L_{\mathrm{X}}-N_{\text {gals. }}$. This may be due to the smaller fraction of clusters excluded based on contrast for the $T_{\mathrm{X}}-N_{\text {gals }}$ relation (see above), such that this relation is already restricted to high-contrast systems. There is still a small difference between the results with different centroids, and $L_{\mathrm{opt}}$ again defines a shallower relation than $N_{\text {gals. }}$. Our findings also show good agreement with the literature results.

A few comments on our results are still necessary. If it is assumed that mass traces optical light and the gas is in hydrostatic 
equilibrium, we expect the mass-luminosity $\left(M / L_{\mathrm{opt}}\right)$ ratio to be constant and the gas temperature to be proportional to mass $\left(T \propto M^{2 / 3}\right)$. The X-ray luminosity is also related to temperature $\left(L_{\mathrm{X}} \propto T^{\alpha}\right)$, although the precise value of $\alpha$ is subject to some debate. The theoretical expectation is $\alpha=2$, but its observed estimate is closer to 3 (this work and David et al. 1993). Considering these relations and assuming $\alpha=3$, for instance, we expect the X-ray and optical luminosities to be related as $\left(L_{\mathrm{X}} \propto L_{\mathrm{opt}}^{2}\right)$ and the relation to temperature to be $T_{\mathrm{X}} \propto L_{\mathrm{opt}}^{0.7}$. If we consider that $N_{\text {gals }}$ is directly proportional to $L_{\text {opt }}$ our results point to steeper relations. $L_{\mathrm{X}} \propto L_{\mathrm{opt}}^{3}$ and $T_{\mathrm{X}} \propto L_{\mathrm{opt}}^{0.8}$, although $T_{\mathrm{X}}-L_{\mathrm{opt}}$ becomes less steep if we exclude clusters with substructure. As pointed out by Donahue et al. (2001) and Popesso et al. (2004), the steepness of the X-ray to optical relations point to the fact that the massluminosity $\left(M / L_{\mathrm{opt}}\right)$ ratio of galaxy clusters is not likely to be constant. This is actually demonstrated by Popesso et al. (2005), who found $M / L \propto M^{0.2}$. We note that this conclusion could not be made if we had considered the bisector solution. Had we used the bisector, we would find $L_{\mathrm{X}} \propto L_{\mathrm{opt}}^{1.80}$, which is consistent with a constant mass-luminosity ratio. In a future paper we plan to investigate in detail this relation $\left(M / L_{\text {opt }}\right)$ and its possible dependence on substructure in clusters.

\section{SUMMARY}

We have compared a list of X-ray galaxy clusters selected from the literature (BAX) to an optical galaxy cluster catalog (NoSOCS). This comparison covers the whole northern hemisphere for $|b|>30^{\circ}$ and spans the redshift range $0.05 \leq z \leq 0.40$. The X-ray and optical centroids show excellent agreement, with a typical X-ray - optical offset of $<0.50 h^{-1} \mathrm{Mpc}\left(\right.$ or $\left.<400^{\prime \prime}\right)$. The overall recovery rate of X-ray clusters in the optical is $81 \%$, with the missing clusters typically poor and/or distant. Thus, NoSOCS efficiently recovers nearly all X-ray luminous clusters from $\operatorname{BAX}\left(L_{\mathrm{X}} \gtrsim 3.2 \times 10^{44} \mathrm{ergs} \mathrm{s}^{-1}\right)$ at all redshifts below $z \sim$ 0.2 . In terms of richness, the recovery rate is $90 \%$ for clusters with $N_{\text {gals }} \gtrsim 25$ out to $z \sim 0.2$, and for all clusters with $N_{\text {gals }} \gtrsim 80$ out to $z \sim 0.3$.

We employed four statistical tests to search for substructure using optical imaging data. These tests were optimized through the minimization of the scatter in the relations between optical and X-ray properties (namely $T_{\mathrm{X}}-N_{\text {gals }}$ ). We have also shown the dependence of richness on the centroid and aperture. We investigated the dependence of substructure results on these parameters, as well as on the cluster contrast, richness, redshift, and magnitude range used for sampling galaxies. The substructure results shown here are based on the largest sample used for this purpose to date (10,190 clusters). As noted by Pinkney et al. (1996), some tests are much more sensitive than others. For instance, the $\beta$, or symmetry, test indicates that $35 \%$ of the clusters have substructure at the $5 \%$ significance level (in a radius of $1.50 h^{-1} \mathrm{Mpc}$ and magnitude range of $m_{r}^{*}-1 \leq m_{r} \leq m_{r}^{*}+1$ ), while the FE test finds $21 \%$, the Lee $2 \mathrm{D}$ test indicates $45 \%$ and AST only $13 \%$.

We have also compared richness $\left(N_{\text {gals }}\right)$ and X-ray cluster properties $\left(L_{\mathrm{X}}\right.$ and $\left.T_{\mathrm{X}}\right)$. We find that $T_{\mathrm{X}}$ correlates better to $N_{\text {gals }}$ than $L_{X}$. We examined the potentially dominant factors affecting these scaling relations. We find that the correlations are most sensitive to the aperture used for estimating richness. For larger radii we sample many more cluster galaxies, but the background noise also becomes much stronger. We find that the optimal radius is $0.50 h^{-1} \mathrm{Mpc}$, which is in good agreement to that found by Popesso et al. (2004). Adopting this radius for estimating
$N_{\text {gals, }}$, we find that the cluster contrast is the dominant source of scatter in the $L_{\mathrm{X}}-N_{\text {gals }}$ relation. The presence of substructure does not affect the scatter of this relation. On the other hand, when comparing $T_{\mathrm{X}}$ to the optical parameters we find that substructure has a strong effect on the scatter.

Our findings also corroborate previous results in the literature, although we note some issues that could explain some of the difficulties in performing these comparisons. These are mainly related to the contrast of the systems, as higher contrast systems generally define steeper relations. For instance, if we compare samples that span different mass regimes, we may find very different correlations, as the contrast cuts will be very different. Relations derived from richness (or $L_{\text {opt }}$ ) calculated from the X-ray centroid have smaller slopes than ones obtained using the optical centroid, due to the smaller galaxy number density seen around the X-ray centroids. The use of different linear regression methods can also lead to inconsistent results, especially for very steep and noisy relations. As shown in $\S 6$, the bisector solution is generally less efficient in recovering the true slopes. Literature results would likely show much better agreement if the same linear regression methods were used. The steeper slopes obtained here also suggest (as have other recent works) that the massluminosity ratio may not be constant. The fitting method and centroid used are crucial for reaching this conclusion. For the $L_{\mathrm{X}}-L_{\mathrm{opt}}$ relation the bisector solution indicates $L_{\mathrm{X}} \propto L_{\mathrm{opt}}^{1.80}$, which is close to the expectations from a constant mass-luminosity ratio.

In the future we plan to use both deeper optical and X-ray data to investigate substructure. These will allow us to assess the reliability of the optical estimates, as well as to investigate the evolution of substructure with look-back time. We also plan to use apertures that scales with mass, such as $R_{500}$ and $R_{200}$ to see whether the structure segregation we show here is also found with these apertures. Such virial radii are more typically used when measuring cluster parameters from large-scale simulations, and it is crucial to use the same techniques in comparing these with observations. Finally, we plan to investigate the massluminosity ratio, as well as estimate the cluster mass function with the NoSOCS data.

The processing of DPOSS and the production of the PalomarNorris Sky Catalog (PNSC) on which this work was based was supported by generous grants from the Norris Foundation, and other private donors. Some of the software development was supported by the NASA AISRP program. We also thank the staff of Palomar Observatory for their expert assistance in the course of many observing runs. We would like to thank the anonymous referee for useful comments. Finally, we acknowledge the efforts of the POSS-II team, and the plate scanning team at STScI. P. A. A. L. was supported by the Fundação de Amparo à Pesquisa do Estado de São Paulo (FAPESP, process 03/04110-3). S. G. D. and A. A. M. were supported in part by the NSF grant AST 0407448. Several undergraduates participated in the data acquisition and processing toward the photometric calibration of DPOSS. P. A. A. L. would like to thank Jason Pinkney for sharing the code used for estimating substructure and for helpful discussions on this code's implementation. This research has made use of the X-Rays Clusters Database (BAX) which is operated by the Laboratoire d'Astrophysique de Tarbes-Toulouse (LATT), under contract with the Centre National d'Etudes Spatiales (CNES). 
Akritas, M., \& Bershady, M. 1996, ApJ, 470, 706

Bahcall, N. A., Fan, X., \& Cen, R. 1997, ApJ, 485, L53

Bahcall, N. A., et al. 2003, ApJ, 585, 182

Basilakos, S., et al. 2004, MNRAS, 351, 989

Blanchard, A., \& Bartlett, J. G. 1998, A\&A, 332, L49

Carlberg, R., Morris, S., Yee, H., \& Ellingson, E. 1997, ApJ, 479, L19

Coleman, G. D., Wu, C. C., \& Weedman, D. W. 1980, ApJS, 43, 393

David, L. P., Slyz, A., Jones, C. Forman, W., Vrtilek, S. D., \& Arnaud, K. A. 1993, ApJ, 412, 479

Donahue, M., Voit, G., Gioia, I., Lupino, G., Hughes, J., \& Stocke, J. 1998, ApJ, 502, 550

Donahue, M., et al. 2001, ApJ, 552, L93 2002, ApJ, 569, 689

Eke, V., Cole, S., Frenk, C.. \& Henry,J. 1998, MNRAS, 298, 1145

Feigelson, E. D., \& Babu, G. J. 1992, ApJ, 397, 55

Fitchett, M. J., \& Webster, R. 1987, ApJ, 317, 653

Fitchett, M. J. 1988, MNRAS, 230, 161

Gal, R. R., de Carvalho, R. R., Lopes, P. A. A., Djorgovski, S. G., Brunner, R. J., Mahabal, A. A., \& Odewahn, S. C. 2003, AJ, 125, 2064

Gilbank, D. 2001, Ph.D. Thesis, Univ. Durham

Gilbank, D. G., Bower, R., Castander, F., \& Ziegler, B. L. 2004, MNRAS, 348, 551 Isobe, T., Feigelson, E. D., Akritas, M., \& Babu, G. J. 1990, ApJ, 364, 104

Jones, C., \& Forman, W. 1999, ApJ, 511, 65

Kitayama, T. \& Suto, Y. 1997, ApJ, 490, 557

Kolokotronis, V., Basilakos, S., Plionis, M., \& Georgantopoulos, I. 2001, MNRAS, 320, 49

Kriessler, J. R., \& Beers, T. C. 1997, AJ, 113, 80
REFERENCES

Lee, K. L. 1979, J. Am. Stat. Assoc., 74, 708

López-Cruz, O., Barkhouse, W., \& Yee, H. 2004, ApJ, 614, 679

Lubin, L. M., Mulchaey, J. S., \& Postman, M. 2004, ApJ, 601, L9

Markevitch, M. 1998, ApJ, 504, 27

Mathiesen, B., \& Evrard, A. E. 1998, MNRAS, 295, 769

Mathiesen, B., Evrard, A. E., \& Mohr, J. J. 1999, ApJ, 520, L21

Mohr, J. J., Evrard, A. E., Fabricant, D. G., \& Geller, M. J. 1995, ApJ, 447, 8

O'Hara, T. B., Mohr, J. J., Bialek, J. J., \& Evrard, A. E. 2006, ApJ, 639, 64

Paolillo, M., Andreon, S., Longo, G., Puddu, E., Gal, R. R., Scaramella, R., Djorgovski, S. G., \& de Carvalho, R. 2001, A\&A, 367, 59

Pinkney, J., Roettiger, K., Burns, J., \& Bird, C. 1996, ApJS, 104, 1

Plionis, M., Basilakos, S., Georgantopoulos, I., \& Georgakakis, A. 2005, ApJ, 622, L17

Popesso, P., Biviano, A., Böhringer, H., Romaniello, M., \& Voges, W. 2005, A\&A, 433, 431

Popesso, P., Böhringer, H., Brinkmann, J., Voges, W., \& York, D. 2004, A\&A, 423, 449

Postman, M., Lauer, T. R., Oegerle, W., \& Donahue, M. 2002, ApJ, 579, 93

Reichart, D. E., et al. 1999, ApJ, 518, 521

Rhee, G. F. R. N., van Haarlem, M. P., \& Katgert, P. 1991a, A\&AS, 91, 513 1991b, A\&A, 246, 301

Rines, K., \& Diaferio, A. 2006, AJ, submitted (astro-ph/0602032)

Smith, G. P., Kneib, Jean-Paul, Smail, I., Mazzotta, P., Ebeling, H., \& Czoske, O. 2005, MNRAS, 359, 417

Voit, G. M. 2005, Rev. Mod. Phys., 77, 207

West, M. J., Oemler, A., \& Dekel, A. 1988, ApJ, 327, 1

Yee, H. K. C. and Ellingson, E. 2003, ApJ, 585, 215 